\title{
International Portfolios: A Comparison of Solution Methods*
}

\author{
Katrin Rabitsch $^{\dagger} \quad$ Serhiy Stepanchuk ${ }^{\ddagger} \quad$ Viktor Tsyrennikov ${ }^{\S}$
}

February 2, 2015

\begin{abstract}
We compare the performance of the perturbation-based (local) portfolio solution method of Devereux and Sutherland $(2010,2011)$ with a global solution method. As a test suite we use model specifications that broadly capture features of international financial trade, between advanced economies, and between advanced and emerging economies. We consider both symmetric country setups and asymmetric setups, that capture important empirical facts such as differences in macroeconomic volatility, differences in portfolio composition, and high equity premia. We find that the local method performs well at business cycle frequencies, both in the symmetric and asymmetric settings, while significant differences arise at long horizons in asymmetric settings.
\end{abstract}

Keywords: Country Portfolios, Solution Methods JEL-Codes: E44, F41, G11, G15

\footnotetext{
${ }^{*}$ We are grateful to Michael B. Devereux, Martin D.D. Evans, Michael Reiter, Alan Sutherland, and seminar and conference participants at the SED 2013, CEF 2013, EEA 2013, and the 2013 European Commission - CEPR workshop on 'Current Account Imbalances and International Financial Integration' for helpful comments.

${ }^{\dagger}$ Vienna University of Economics and Business. E-mail: katrin.rabitsch@wu.ac.at.

${ }^{\ddagger}$ École Polytechnique Fédérale de Lausanne. E-mail: serhiy.stepanchuk@epfl.ch

${ }^{\S}$ Cornell University. E-mail: viktor.tsyrennikov@gmail.com
} 


\section{Introduction}

This paper presents and evaluates two solution methods for computing optimal portfolios in dynamic stochastic general equilibrium (DSGE) settings with incomplete markets. The first is the perturbation-based local method of Devereux and Sutherland (2010, 2011) (hereafter DS). This method, as its name suggests, guarantees desired accuracy locally, that is, in the vicinity of the approximation point (the non-stochastic steady state). The second is the global solution method as implemented in Stepanchuk and Tsyrennikov (2014). Global methods can achieve desired accuracy throughout the state space. This comes at the cost of having to use a non-linear equation solver that adds significantly to computation time. Global methods also require more significant investment in approximation techniques if the dimensionality of the endogenous state space is large. ${ }^{1}$ The DS method is faster, but its main advantage is that it builds upon the widely-used toolkit of a macroeconomist - a set of algorithms to compute the first and the second-order approximations to solutions of dynamic stochastic general equilibrium models, e.g. Schmitt-Grohé and Uribe (2003). Thus, it is relatively easy to apply in macroeconomic models extended to include a non-trivial portfolio choice. It provides easy-to-interpret expressions for optimal constant portfolios, referred to as steadystate portfolios, and for first-order portfolio dynamics. Available analytical partial results are helpful in building intuition about mechanisms at work. ${ }^{2}$

The DS method has been widely adopted by the international macroeconomics and finance literature that until recently had ignored facts related to portfolio size and composition. ${ }^{3,4}$ Early models of international capital flows featured either incomplete financial markets with only one asset or complete financial markets. In the former case only net capital flows could be analyzed. In the latter case portfolios are constant and capital flows are absent unless preferences are time non-separable as shown by Judd et al (2003). Moreover, financial trade is often cast in terms of fictitious Arrow securities that could not be linked to real assets. The DS method applies to models with both complete and incomplete markets and allows for arbitrary asset market structures. This methodological progress has allowed researchers to address the vast changes in the international financial landscape during the recent decades: the emergence and rapid growth of gross external positions, growing two-way capital flows, the role of portfolio re-balancing in determining net capital flows, and the potential influences of size and composition of gross portfolios on macroeconomic outcomes themselves through

\footnotetext{
${ }^{1}$ A series of solution methods developed by Judd et al. (2012) make it increasingly feasible to solve medium to large-scale macroeconomic models. For example, Judd et al. (2011) demonstrate how to solve a 60-country model without resorting to powerful computation assets.

${ }^{2}$ Because of their relative ease of implementation local solution methods are a default choice when it comes to solving medium- and large-scale DSGE models. Recent market developments, however, prove the importance to include into macroeconomic models occasionally binding constraints such a zero lower bound on the interest rate or financial constraints. These constraints can be handled effectively only by global solution methods that are increasingly often chosen to solve medium-scale models.

${ }^{3}$ Among others see Devereux and Sutherland (2008), Devereux and Sutherland (2009), Coeurdacier et al. (2010), Devereux and Yetman (2010), Devereux and Sutherland (2010c), Devereux and Sutherland (2010b), Amdur (2010), Coeurdacier and Gourinchas (2011), Viani (2011), Nguyen (2011), Gertler et al. (2012), Benigno and Nistico (2012), Berriel and Bhattarai (2013), Berriel (2013), and Karadi et al. (2013).

${ }^{4}$ The DS method is not the only alternative. Concurrently, Tille and van Wincoop (2007) described an alternative implementation of the same solution method. A different hybrid approach is developed by Evans and Hnatkovska $(2005,2012)$ who utilize closed-form portfolio solutions from continuous-time portfolio choice models. Judd and Guu (2001) appeal to bifurcation theory to derive a Taylor-series approximation to the optimal portfolio in a static setting.
} 
exchange rate and asset price driven valuation effects.

Despite its wide-spread adoption, little is known about the accuracy of the DS solution method and, therefore, the 'domain' of its applicability. Our paper tries to fill this gap. To this end, we perform a comprehensive evaluation of the method against the global solution method. We compare solutions along the following dimensions: policy functions and Euler equation errors, simulated short time paths and moments, ergodic moments and wealth distributions, and welfare measures.

Our laboratory for the evaluation of the two methods is a two country model with labor and capital income endowments and an array of traded financial assets that includes domestic and foreign equities and a bond. Our test suite then consists of two special cases of this general model structure. The key difference between the two specifications is that in the first all assets pay nearly the same expected return, while in the second equities pay a sizeable risk premium.

Model specification 1 shuts down the bond market, becoming the setting analyzed in Devereux and Sutherland (2011). We analyze both symmetric and asymmetric economies. We think of the symmetric setting as modeling financial trade between similar advanced economies, e.g. France and the U.K. The DS method performs extremely well in this case, both at business cycle and at medium to long-run frequencies. We think of the asymmetric setting as modeling financial trade between advanced and emerging market economies, e.g. the U.S. and Brazil. The defining feature of emerging market economies is higher macroeconomic volatility as documented in Aguiar and Gopinath (2007). To capture this asymmetry we assume that the foreign country's shocks are twice as volatile as those of the home country. We find that the DS method continues to perform well at business cycle frequencies, but that one needs to be cautious when using the method to characterize long-term (ergodic) properties of the model. This is a consequence of the fact that model characteristics such as cross-country differences in shock volatilities fail to pin down the aggregate wealth distribution (the net foreign asset position) at the approximation point of the deterministic steady state. This problem is frequently addressed by introducing a stationarity-inducing device, such as the endogenous discount factor (EDF). When comparing local and global solution method with inclusion of an EDF, we find that both solutions give rise to very similar results, even in terms of ergodic model properties. The EDF, however, strongly affects the model solution and dominates any economic forces that would otherwise lead to asymmetric ergodic NFA distributions. We demonstrate this in a version where the EDF is replaced by a borrowing limit under the global method: the stationary distribution in this case looks very different and the asymmetries are well reflected in it. So, we ask if in asymmetric settings the local solution can be improved upon by an appropriate choice of the approximation point. We find that iterative updating of an approximation point, as suggested by Devereux and Sutherland (2009), yields unsatisfactory results. Using the mean NFA computed under the global solution provides us with the most accurate solution, yet it still leads to no improvement over using the symmetric deterministic steady state. We also discuss the relation to the 'risky steady state' literature, see Coeurdacier et al. (2011), Julliard (2011) and De Groot (2013). At a risky steady state returns of different traded assets are generally different from each other in asymmetric settings. We document that this precludes direct application of the DS solution formulas; so, we do not consider it.

Model specification 2 aims to address deeper asymmetries, that are key in capturing empirical observations of the financial trade between advanced and emerging market economies. The largest part of financial globalization has taken place in and between advanced economies. 
However, the recent growth experience of large emerging economies, particularly large BRIC (Brazil, Russia, India, China) countries, means that emerging countries now also play a significant role in international financial markets. ${ }^{5}$ Advanced and emerging market economies display strong structural differences in the amount of risk these country groups face, be it because of different macroeconomic volatility they face or because of differences in financial market development as in Mendoza et al. (2009a). This could also be a result of the limited supply of safe assets in the emerging market economies, as emphasized by Caballero et al. (2006). The above mentioned economic differences result in heterogeneous NFA positions and compositions of external portfolios. Gourinchas and Rey (2013) document that advanced economies typically have a much higher share of 'risky' assets - defined as portfolio equity or FDI - on their asset side of the balance sheet than emerging economies. ${ }^{6}$ They also compute the net 'risky' position, the share of risky asset in the asset side of the external balance sheet minus the share of risky assets on the external liability side. The G7 economies are increasingly long in net risky assets, while the BRIC economies, particularly in the 1990s, have increasingly taken net short positions in risky assets: in 2010 the G7 economies' net risky position stands at around 15\% of GDP, and the BRIC economies' position at around $-30 \%$ of GDP, compared to being below plus and minus five percent in 1990 respectively. Most prominently, and contrary to neoclassical wisdom, the U.S. emerged as the world largest debtor that arguably holds the riskiest portfolio, earning a higher return on its assets than the rest of the world. Gourinchas and Rey (2007a) have coined the expression of the U.S. having become the world venture capitalist for this phenomenon.

Our model specification 2 aims to capture, in a stylized setting, such key 'financial' aspects of asymmetries in risk taking and a sizable equity premium ('excess return') in the simplest possible way. In this setup, the two economies can trade a risk-free bond and a risky claim to the foreign country's capital income endowment. We allow risk attitudes to differ across countries as in Gourinchas et al. (2010) and Stepanchuk and Tsyrennikov (2014). ${ }^{7}$ We parameterize the model to obtain an equity premium comparable to that observed in the data. Diverse risk attitudes translate into different willingness to hold the risky and the safe asset. The less risk-averse (advanced) economy is more willing to hold the high risk/high return equity: so, it buys a larger share of the risky equity and sells the safe bond. The excess return that the less risk-averse country earns on average, allows it to accumulate net claims on the more risk-averse foreigners. Both the global and the local solution method capture this effect well and generate equity premia that are close in magnitude. But the DS method somewhat understates the holdings of the risky asset. Therefore, under the local solution method wealth accumulation by the less risk-averse economy is also understated. But the difference is small,

\footnotetext{
${ }^{5} \mathrm{~A}$ widely used measure of de facto cross-border financial integration is the sum of external assets and external liabilities, scaled by GDP. Figure B.1 in the appendix documents the development of this measure for advanced economies, for emerging markets economies, for the US, the BRIC economies, and China, based on the external wealth of nations dataset of Lane and Milesi-Ferretti (2001, 2007). Cross-border financial positions for advanced countries increased from $68.3 \%$ in 1980 to $463.2 \%$ in 2007 . For emerging economies this measure of financial integration stood at $50.11 \%$ percent in 1980, and increased to $192.4 \%$ percent in 2007 . Certain countries of the emerging economies group experienced particularly noteworthy increases in financial integration. E.g. for China this measure increased almost tenfold from $15.1 \%$ in 1981 to $113.5 \%$ in 2007.

${ }^{6}$ The share of 'risky' assets is $49 \%$ for the United States, $50 \%$ for Canada, $26 \%$ for the UK, and $31 \%$ for France. In contrast, India's share of 'risky' assets stands at 5\%, Indonesia's at 5\%, Russia's at 18\%, China's at $9 \%$ and Brazil's at $21 \%$.

${ }^{7}$ Differences in attitudes toward risk can be thought of as a short-cut for a different ability to diversify idiosyncratic risk. Maggiori (2013) endogenizes differences in risk-aversion.
} 
and our assessment is that, along short time paths, the DS method performs well. When characterizing the model's ergodic properties, the global and the DS method obtain very different results. We reiterate on our finding from model specification 1: for this purpose, the DS method, being a local method, should be used with caution only.

Finally, we use model specification 2 to highlight the independence of the DS portfolio solution of the size of shocks. The true solution does depend on the shock size, as documented in Rabitsch and Stepanchuk (2014) using a static setting. This inaccuracy shows up in the solution to model specification 2 . The errors in the gross portfolio positions, when multiplied by a significant risk-premium, lead to a misstatement of the valuation effect that feeds into current account dynamics.

The paper is organized as follows. Section 2 describes the general model framework. Section 3 discusses the local (DS) and global solution methods. Section 4 describes results from model specification 1 with both symmetric (4.1) and asymmetric (4.2) countries. We discuss the issues related to the choice of the approximation point in section 4.2.2. Section 5 discusses results from model specification 2 that considers more fundamental asymmetries between the two model economies, and a pronounced return differential. Section 6 concludes.

\section{Model}

Here we describe the general features of the model that we consider in this paper, and later (in the corresponding calibration sections) discuss features and restrictions specific to model specifications 1 and 2. The model consists of two countries, labeled $h$ and $f$. The representative agent of each country has preferences over a single consumption good. We abstract from modeling a production side, and assume that instead output arrives exogenously. In particular, uncertainty in the model is represented by four exogenous stochastic processes: $\left\{Y_{h t}^{k}, Y_{h t}^{l}, Y_{f t}^{k}, Y_{f t}^{l}\right\} \equiv \mathbf{Y}_{t}$. They model home capital income, home labor income, foreign capital income and foreign labor income. All of the above are first-order autoregressive processes:

$$
\begin{aligned}
& \log \left(Y_{h t}^{k} / \bar{Y}_{h}^{k}\right)=\rho_{h}^{k} \log \left(Y_{h t-1}^{k} / \bar{Y}_{h}^{k}\right)+\varepsilon_{h t}^{k}, \\
& \log \left(Y_{h t}^{l} / \bar{Y}_{h}^{l}\right)=\rho_{h}^{l} \log \left(Y_{h t-1}^{l} / \bar{Y}_{h}^{l}\right)+\varepsilon_{h t}^{l}, \\
& \log \left(Y_{f t}^{k} / \bar{Y}_{f}^{k}\right)=\rho_{f}^{k} \log \left(Y_{f t-1}^{k} / \bar{Y}_{f}^{k}\right)+\varepsilon_{f t}^{k}, \\
& \log \left(Y_{f t}^{l} / \bar{Y}_{f}^{l}\right)=\rho_{f}^{l} \log \left(Y_{f t-1}^{l} / \bar{Y}_{f}^{l}\right)+\varepsilon_{f t}^{l},
\end{aligned}
$$

where $\left\{\varepsilon_{h t}^{k}, \varepsilon_{h t}^{l} \varepsilon_{f t}^{k}, \varepsilon_{f t}^{l}\right\}$ is a vector of i.i.d. innovations with zero mean, finite support and variance-covariance matrix $\Sigma_{\varepsilon}$. Aggregate output in country $a \in\{h, f\}$ is the sum of capital and labor income endowments: $Y_{a t} \equiv Y_{a t}^{k}+Y_{a t}^{l}$.

The representative agent in country $a \in\{h, f\}$ values different consumption plans $\left\{c_{a \tau}\right\}_{\tau=t}^{\infty}$ according to the Epstein-Zin utility function:

$$
V_{a t} \equiv \max _{c_{a t}}\left[\left(1-\beta\left(\bar{c}_{a t}\right)\right) c_{a t}^{\frac{1-\gamma_{a}}{\lambda_{a}}}+\beta\left(\bar{c}_{a t}\right)\left(E_{t} V_{a t+1}^{\left(1-\gamma_{a}\right)}\right)^{\frac{1}{\lambda_{a}}}\right]^{\frac{\lambda_{a}}{1-\gamma_{a}}}
$$

where $c_{a t}$ is consumption, $\beta\left(\bar{c}_{a t}\right)$ is the endogenous discount factor, $\bar{c}_{a t}$ is the average consumption in country $a, \gamma_{a}$ is the coefficient of risk-aversion, while $\lambda_{a}=\frac{1-\gamma_{a}}{1-\frac{1}{\psi_{a}}}$ with $\psi_{a}$ being 
the intertemporal elasticity of substitution. When $\lambda_{a}=1$, we get as a special case the time-additive expected utility CRRA preferences.

Financial markets trade claims to home and foreign capital income streams, as well as a one-period risk-free discount bond which pays one unit of consumption good and is in zero net supply. Let $q_{b t}$ be the price of this bond. Let $q_{a t}$ be the price of a claim to a stream of capital income $\left\{Y_{a \tau}^{k}\right\}_{\tau=t}^{\infty}$ produced in country $a \in\{h, f\}$. These prices will be sometimes referred to as countries' stock market indices. The representative agent in country $a$ then maximizes his life-time utility (5) subject to the following budget constraint:

$$
c_{a t}+\theta_{h t}^{a} q_{h t}+\theta_{f t}^{a} q_{f t}+b_{t}^{a} q_{t}^{b}=\theta_{h t-1}^{a}\left(q_{h t}+Y_{h t}^{k}\right)+\theta_{f t-1}^{a}\left(q_{f t}+Y_{f t}^{k}\right)+b_{t-1}^{a}+Y_{a t}^{l},
$$

where $\theta_{h t}^{a}, \theta_{f t}^{a}$ and $b_{t}^{a}$ denote country $a$ 's purchases of domestic and foreign equity claims and the bond.

When looking at different model specifications in our test suite, we impose further restrictions on the general financial market setup just described. In model specification 1, this bond market is shut down, which can formally be achieved by additionally assuming a zeroborrowing limit $^{8}$, which together with the market-clearing condition will imply that both investors' bond positions are always equal to 0 . In model specification 2, we assume that there is trade in the one-period risk-free discount bond, $b_{t}^{a}$, but, for simplicity, shut down the domestic equity market.

The goods market clearing condition is:

$$
c_{h t}+c_{f t}=Y_{h t}+Y_{f t} .
$$

Asset markets clearing conditions are:

$$
\begin{aligned}
\theta_{h t}^{h}+\theta_{h t}^{f} & =1, \\
\theta_{f t}^{h}+\theta_{f t}^{f} & =1, \\
b_{t}^{h}+b_{t}^{f} & =0 .
\end{aligned}
$$

This completes the description of our general model structure on which the methods comparison is based. An issue that, however, deserves further explanation is our model choice of including an endogenous discount factor (EDF). The discount factor function $\beta$ : $R_{+} \rightarrow[0,1)$ is non-increasing. As is well-known, if $\beta($.$) is a constant function and financial$ markets are incomplete, then in a local solution that is based on a first-order Taylor series approximation, countries' net financial positions are non-stationary: the unit root in wealth dynamics implies that the solution allows reaching financial positions that are known to be infeasible. Schmitt-Grohé and Uribe (2003) propose several stationarity-inducing devices to remedy this situation, one of which is the EDF. We follow Devereux and Sutherland (2011, $2010,2009)$ in choosing the precise functional form, given by $\beta(c)=\beta c^{-\eta}$.

When solving the model with the global method, the endogenous discount factor is not required. To obtain a well defined solution in such case, it suffices to assume the presence of a borrowing constraint. A borrowing constraint can be specified very loosely, e.g. as loose as the natural borrowing constraint, such that the constraints become binding only in rare cases, or it can be specified as a somewhat tighter, ad-hoc constraint. To do a comparison of

\footnotetext{
${ }^{8}$ Formally, $b_{t}^{a} \geq 0$ where $b_{t}^{a}$ is country $a$ 's position in the bond.
} 
local and global solution methods justice, it would not be 'fair' to compare the local solution with a global solution in which a borrowing constraint binds frequently. We compare the local solution to two versions of the global solution method. In the first, for sake of direct comparability, we abstract entirely from a borrowing constraint and introduce the endogenous discount factor also when the model is solved with the global method. This setting allows for the cleanest comparison of local and global solution methods, as we subject the solution methods to an exactly identical model structure. In the second, we also present a version of the global solution that dispenses the EDF and instead specifies an ad-hoc borrowing constraint. We do so because we find that the presence of the EDF can itself have a distortive impact on the solution. In particular, in asymmetric setups, the presence of the EDF mutes the effects of (differential) precautionary motives that would otherwise lead to asymmetries in the ergodic distribution of net foreign assets, and induces a relatively symmetric distribution. The budget constraint in the borrowing limits case takes the following form:

$$
\theta_{h t-1}^{h}\left(q_{h t}+Y_{h t}^{k}\right)+\theta_{f t-1}^{h}\left(q_{f t}+Y_{f t}^{k}\right)+b_{t}^{h} \geq B L, \quad B L \in \mathbb{R} .
$$

In the remainder of the paper (in all figures and tables), we report as 'global' the results from the solution of the global method that employs the EDF, and report as 'global BL' the results from the solution of the global method with borrowing limits.

\section{Global and local solution methods}

In the following we provide a description of global and local numerical solution methods ${ }^{9}$.

\subsection{Global solution method}

The equilibrium is characterized by a system of first order and equilibrium conditions, which include the value function definition, first-order optimality conditions with respect to consumption and asset choices (Euler equations), budget constraints and market-clearing conditions. It is summarized in table 1.

The 'natural' state space for our model includes portfolio positions of each country. Following Kubler and Schmedders (2003) and Stepanchuk and Tsyrennikov (2011), we recast the equilibrium conditions in a form that is consistent with a wealth-recursive equilibrium. This allows us to reduce the dimensionality of the problem, as the wealth share, $\omega_{t}$, becomes the model's only endogenous state variable. More precisely, this transformed state variable expresses the domestic country's financial wealth share in total (world) financial wealth, which, for the case explicit borrowing constraints are absent, can be written as:

$$
\omega_{t}=\frac{\theta_{h t-1}^{h}\left(q_{h t}+Y_{h t}^{k}\right)+\theta_{f t-1}^{h}\left(q_{f t}+Y_{f t}^{k}\right)+b_{t}^{h}+Y_{h t}^{l}}{q_{h t}+Y_{h t}+q_{f t}+Y_{f t}} .
$$

$\omega_{t}=0.5$ corresponds to the case where total financial wealth is divided evenly between the two countries. Unlike Kubler and Schmedders (2003) and Stepanchuk and Tsyrennikov

\footnotetext{
${ }^{9}$ We use the general model here to describe the solutions methods. In practice, we do a few small changes to the algorithm when we apply it to models specifications 1 and 2: (1) we drop the bond market-clearing conditions and the bond Euler equations in model specification 1; (2) we make the appropriate changes to the equity market-clearing conditions and equity Euler equations in model specification 2.
} 


$$
\begin{array}{ll}
\text { (A1): } & V_{h t}=\max _{c_{h t}}\left[\left(1-\beta\left(c_{h t}\right)\right) c_{h t}^{\frac{1-\gamma_{h}}{\lambda_{h}}}+\beta\left(c_{h t}\right)\left(E_{t} V_{h t+1}^{\left(1-\gamma_{h}\right)}\right)^{\frac{1}{\lambda_{h}}}\right]^{\frac{\lambda_{h}}{1-\gamma_{h}}}, \\
\text { (A2): } & V_{f t}=\max _{c_{f t}}\left[\left(1-\beta\left(c_{f t}\right)\right) c_{f t}^{\frac{1-\gamma_{f}}{\lambda_{f}}}+\beta\left(c_{f t}\right)\left(E_{t} V_{f t+1}^{\left(1-\gamma_{f}\right)}\right)^{\frac{1}{\lambda_{f}}}\right]^{\frac{\lambda_{f}}{1-\gamma_{f}}}, \\
\text { (A3): } & M_{h t+1}=\frac{\partial V_{h t} / \partial c_{h t+1}}{\partial V_{h t} / \partial c_{h t}}, \\
\text { (A4): } & M_{f t+1}=\frac{\partial V_{f t} / \partial c_{f t+1}}{\partial V_{f t} / \partial c_{f t}} \\
\text { (A5): } & q_{h t}=\beta\left(c_{h t}\right) E_{t} M_{h t+1}\left(q_{h t+1}+Y_{h t+1}^{k}\right), \\
\text { (A6): } & q_{f t}=\beta\left(c_{h t}\right) E_{t} M_{h t+1}\left(q_{f t+1}+Y_{f t+1}^{k}\right), \\
\text { (A7): } & q_{b t}=\beta\left(c_{h t}\right) E_{t} M_{h t+1}, \\
\text { (A8): } & q_{h t}=\beta\left(c_{f t}\right) E_{t} M_{f t+1}\left(q_{h t+1}+Y_{h t+1}^{k}\right), \\
\text { (A9): } & q_{f t}=\beta\left(c_{f t}\right) E_{t} M_{f t+1}\left(q_{f t+1}+Y_{f t+1}^{k}\right), \\
\text { (A10): } & q_{b t}=\beta\left(c_{f t}\right) E_{t} M_{f h t+1}, \\
\text { (A11): } & c_{h t}+c_{f t}=Y_{h t}+Y_{f t}, \\
\text { (A12): } & c_{h t}+\theta_{h t}^{h} q_{h t}+\theta_{f t}^{h} q_{f t}+b_{t}^{h} q_{b t}=\theta_{h t-1}^{h}\left(q_{h t}+Y_{h t}^{k}\right)+\theta_{f t-1}^{h}\left(q_{f t}+Y_{f t}^{k}\right)+b_{t-1}^{h}+Y_{h t}^{l}, \\
\text { (A13): } & \theta_{h t}^{h}+\theta_{h t}^{f}=1 \\
\text { (A14): } & \theta_{f t}^{h}+\theta_{f t}^{f}=1, \\
\text { (A15): } & b_{t}^{h}+b_{t}^{f}=0 .
\end{array}
$$

Table 1: System of equilibrium conditions.

(2011), when we do not impose borrowing limits and short-sale constraints, nothings guarantees that $\omega_{t} \in[0,1]$. However, in practice, in all our simulations $\omega_{t}$ remains between 0 and 1 with very high probability. In our numerical algorithm, we choose a grid for $\omega_{t}$ to cover the interval of $[-0.5,1.5]$, and we extrapolate when the realized $\omega_{t}$ falls outside of this interval using a quadratic extension of the computed equilibrium policy functions.

When solving the model under the presence of a borrowing constraint, the domestic country's financial wealth in total (world) financial wealth instead is:

$$
\omega_{t}=\frac{\theta_{h t-1}^{h}\left(q_{h t}+Y_{h t}^{k}\right)+\theta_{f t-1}^{h}\left(q_{f t}+Y_{f t}^{k}\right)+b_{t}^{h}+Y_{h t}^{l}-B L}{\left(q_{h t}+Y_{h t}+q_{f t}+Y_{f t}-2 B L\right)},
$$

where parameter $B L$ ('borrowing limit') governs the degree to which countries can short-sell their equity, which determine the tightness of the countries' borrowing limits. The assumption of short-selling constraints is common in the literature of global portfolio solution methods and is generally used to insure that the wealth share can take on only values in the interval $[0,1]$. In principle, a constraint on the maximum amount of short-selling allowed can be placed on either individual holdings of equity positions (i.e. on $\theta_{h t}^{h}$ and $\theta_{f t}^{h}$ individually, and on $\theta_{h t}^{f}$ and $\theta_{f t}^{f}$ individually) or could be placed on the value of the joint equity position (i.e., that $\theta_{h t-1}^{h}\left(q_{h t}+Y_{h t}^{k}\right)+\theta_{f t-1}^{h}\left(q_{f t}+Y_{f t}^{k}\right)+b_{t}^{h} \geq B L$, and similarly for Foreign). ${ }^{10}$ Since we want to compare the global solution with the local solution (DS method) that ignores such short-selling constraints, we need to insure that our constraints are not too tight.

Using the definition in (11) (respectively, (12)), we can rewrite the budget constraint of

\footnotetext{
${ }^{10}$ For example, an assumption often made is a no-short-selling constraint on (individual) equity positions, such that $\theta_{h t}^{h}, \theta_{f t}^{h}, \theta_{h t}^{f}, \theta_{f t}^{f} \geq 0$.
} 
the home economy in the system of equilibrium conditions as:

$$
c_{h t}+\theta_{h t}^{h} q_{h t}+\theta_{f t}^{h} q_{f t}+b_{t}^{h} q_{h t}=\left(q_{h t}+Y_{h t}+q_{f t}+Y_{f t}\right) \omega_{t} .
$$

Let $\mathbf{Y}$ and $\omega$ denote respectively current, date $t$, values of the exogenous income states and the wealth share, and $\mathbf{Y}^{\prime}$ and $\omega^{\prime}$ denote their next-period values. In a wealth-recursive equilibrium, equilibrium functions (consumption and portfolio policies, pricing and value functions) depend only on $\mathbf{Y}$ and $\omega$. Let $\rho(\omega, \mathbf{Y})$ denote the vector of these equilibrium functions. We approximate these functions by cubic splines. To solve for the spline coefficients, we use a time-iteration collocation algorithm similar to Kubler and Schmedders (2003) and Stepanchuk and Tsyrennikov (2011). We start with some initial guess $\rho^{0}$. During each iteration, we use the spline coefficients of $\rho^{n}$ from the previous iteration, and for each $(\omega, \mathbf{Y})$ on some fixed grid, we solve for the prices and optimal consumption and portfolio choices that satisfy the system of equilibrium conditions. In particular, we simultaneously solve two nested systems of non-linear equations. Given the portfolio choice of the home country, $\left(\theta_{h}^{h}, \theta_{f}^{h}, b^{h}\right)$, the next-period wealth share is implicitly defined by:

$$
\omega^{\prime}=\frac{\left(q_{h}\left(\omega^{\prime}, \mathbf{Y}^{\prime}\right)+Y_{h}^{k \prime}\right) \theta_{h}^{h}+\left(q_{f}\left(\omega^{\prime}, \mathbf{Y}^{\prime}\right)+Y_{f}^{k \prime}\right) \theta_{f}^{h}+Y_{h}^{l \prime}}{q_{h}\left(\omega^{\prime}, \mathbf{Y}^{\prime}\right)+Y_{h}^{\prime}+q_{h}\left(\omega^{\prime}, \mathbf{Y}^{\prime}\right)+Y_{f}^{\prime}},
$$

For any $\left(\theta_{h}^{h}, \theta_{f}^{h}, b^{h}\right)$, we can solve equation (14) for all possible realizations of $\mathbf{Y}$ to find a vector of $\omega^{\prime}(\mathbf{Y})$ 's. Combined with the spline coefficients of equilibrium policy and pricing functions from the previous iteration, this relates current portfolio choices to the future (next-period) dynamics. With this relationship at hand, we can solve equilibrium system of equations in table 1.

Our algorithm can be summarized as follows:

1. Choose a stopping criterion $\delta$, a finite grid for $\omega$ and an initial guess for the equilibrium policy and pricing functions $\rho^{0}$.

2. Given an approximation to the equilibrium policy and pricing functions $\rho^{n}$ from the previous iteration, for each value of $(\omega, \mathbf{Y})$ on the predetermined grid we simultaneously solve (14) and the system of equilibrium conditions in table 1.

3. Compute the spline coefficients of the new approximation to the equilibrium functions, $\rho^{n+1}$.

4. Check if $\left\|\rho^{n+1}-\rho^{n}\right\|<\delta$. If true, terminate the algorithm. If not, increase $n$ by 1 and continue to step (2).

We choose 81 discretization points for $\mathbf{Y}$, three values for each element of the vector. We discretize the VAR process given in (1) as in Lkhagvasuren and Gospodinov (2011). ${ }^{11}$ Finally, we choose 51 discretization points for our endogenous state variable, $\omega$.

\footnotetext{
${ }^{11} \mathrm{~A}$ number of recent papers have shown that the widely used discretization approach described in Tauchen and Hussey (1991) can perform rather poorly when the number of discretization nodes is low or when underlying processes are very persistent: (Flodén (2006), Kopecky and Suen (2010)). For this reason we avoid using the Hussey-Tauchen procedure.
} 


\subsection{Local solution method}

To obtain a local (perturbation) solution, we follow the method of Devereux and Sutherland (2011), henceforth DS. The DS method provides readily applicable solution formulas for the zero-order and first-order parts of an approximation to portfolio holdings, and has, because of its user-friendliness become widely used in recent contributions in macroeconomics. Other noteworthy contributions to solving portfolios with local approximation methods are Samuelson (1970), Judd and Guu (2001), Tille and van Wincoop (2007), and Evans and Hnatkovska (2005)). ${ }^{12}$ The DS perturbation solution method is straightforward to implement and in simple settings it is possible to obtain an analytic characterization of the approximate portfolio solution, which can be helpful for building intuition for the mechanisms at play. Its main advantage is that it can be used in rich models, in the presence of several (endogenous) state variables.

We begin by re-stating the budget constraint of the home country as follows:

$$
\left(\theta_{h t}^{h}-1\right) q_{h t}+\theta_{f t}^{h} q_{f t}+b_{t}^{h} q_{b t}=\left(\theta_{h t-1}^{h}-1\right)\left(q_{h t}+Y_{h t}^{k}\right)+\theta_{f t-1}^{h}\left(q_{f t}+Y_{f t}^{k}\right)+b_{t-1}+Y_{t}-c_{h t} .
$$

Let $\left(\alpha_{h t}^{h}, \alpha_{f t}^{h}, \alpha_{b t}^{h}\right)=\left(\left(\theta_{h t}^{h}-1\right) q_{h t}, \theta_{f t}^{h} q_{f t}, b_{t}^{h} q_{b t}\right)$ be net funds invested in home and foreign equity claims by the home country. ${ }^{13}$ Net funds invested by the foreign country are: $\left(\alpha_{h t}^{f}, \alpha_{f t}^{f}, \alpha_{b t}^{f}\right)=$ $\left(\theta_{h t}^{f} q_{h t},\left(\theta_{f t}^{f}-1\right) q_{f t}, b_{t}^{f} q_{b t}\right)$. The asset market clearing conditions (5) are then replaced by:

$$
\begin{gathered}
\alpha_{h t}^{h}+\alpha_{h t}^{f}=0, \\
\alpha_{f t}^{h}+\alpha_{f t}^{f}=0, \\
\alpha_{b t}^{h}+\alpha_{b t}^{f}=0 .
\end{gathered}
$$

We can write the budget constraint of the home country in terms of $\alpha$ 's:

$$
\alpha_{h t}^{h}+\alpha_{f t}^{h}+\alpha_{b t}^{h}=\alpha_{h t-1}^{h} r_{h t}+\alpha_{f t-1}^{h} r_{f t}+\alpha_{b t-1} r_{b t}+Y_{h t}-c_{h t},
$$

and asset returns:

$$
r_{h t}=\frac{q_{h t}+Y_{h t}^{k}}{q_{h t-1}}, \quad r_{f t}=\frac{q_{f t}+Y_{f t}^{k}}{q_{f t-1}}, \quad r_{b t}=\frac{1}{q_{b t-1}} .
$$

The net foreign asset (NFA) position of country $h$ then evolves according to the following law of motion:

$$
\begin{aligned}
W_{h t} & \equiv \alpha_{h t}^{h}+\alpha_{f t}^{h}+\alpha_{b t}^{h} \\
& =r_{b t} W_{h t-1}+\alpha_{h t-1}^{h}\left(r_{h t}-r_{b t}\right)+\alpha_{f t-1}^{h}\left(r_{f t}-r_{b t}\right)+Y_{h t}-c_{h t} .
\end{aligned}
$$

\footnotetext{
${ }^{12}$ The DS method relates to these other contributions in the following ways. In particular, it builds up on and extends the principles developed by Samuelson (1970) to a dynamic general equilibrium setting. The zero-order (steady state) solution of portfolio holdings obtained by the DS method is equivalent to the zero-order portfolio solution obtained by Judd and Guu's (2001) bifurcation approach to solving portfolios - yet, the Judd and Guu approach is not directly applicable to a dynamic setting. The DS solution method delivers an equivalent solution (for zero- and first-order portfolio holdings) as the iterative method by Tille and van Wincoop (2007). Finally, the DS method is quite different from Evans and Hnatkovska (2005), who combine perturbation methods with continuous-time approximations.

${ }^{13}$ Net is relative to a portfolio of one unit of domestic equity and zero units of other claims. This is the convention used by DS.
} 
The NFA position of the foreign country is $W_{f t}=-W_{h t}$. The solution - the country's policy functions and the price system - are functions of $W_{h t}$ and exogenous shocks $\mathbf{Y}$. This is equivalent to using the home country's wealth share, $\omega_{t} .{ }^{14}$

Generally, applying a perturbation method to a model where agents face a portfolio choice is non-trivial. The reason is that the approximation point is typically chosen to be the solution to a deterministic version of a model. But in a deterministic setting all assets must yield the same return and thus are perfect substitutes. As a consequence, there is a continuum of solutions to a deterministic version as first emphasized in Judd and Guu (2001). A related but distinct difficulty is that, because of certainty equivalence, the portfolio is also indeterminate in a first-order approximation to the model. DS show how to overcome these problems: they solve for the zero-order component of the portfolio solution by combining a first-order approximation to the 'macroeconomic part' of the model with a second-order approximation to the 'portfolio part', Euler equations A1-A4. A second-order approximation to Euler equations and a first-order approximation to the macroeconomic part are in general interdependent. But DS show that this simultaneous system can be used to obtain an analytical solution for the steady-state portfolios, denoted $\alpha(\bar{x})$ in equation (17) below. Similarly, to solve for the firstorder portfolio dynamics, $\alpha_{x}(\bar{x})$, one should combine a second-order approximation to the 'macroeconomic part' with a third-order approximation to Euler equations. One can then write the approximate portfolio solution as:

$$
\alpha_{t} \simeq \alpha(\bar{x})+\alpha_{x}(\bar{x}) \widehat{x}_{t}
$$

In the above expression, $\widehat{x}_{t}$ denotes the vector of state variables, in terms of percentage deviations from steady state (apart from NFA which is in terms of absolute deviations), and let a variable with a bar refer to (deterministic) steady states of that variable. DS also state that their solution principle, which builds up on earlier work by Samuelson (1970), could be successively applied to higher orders: to obtain an $n$-th order accurate portfolio solution, one needs to approximate the portfolio optimality conditions up to order $n+2$, in conjunction with an approximation to the model's other optimality and equilibrium conditions of order $n+1$. E.g., going one order higher, one would obtain the approximate portfolio solution as $\alpha_{t}=\bar{\alpha}+\alpha_{x} \widehat{x}_{t}+\frac{1}{2} \widehat{x}_{t}^{\prime} \alpha_{x x} \widehat{x}_{t}$.

It is important to realize that the expression in equation (17) is, however, not the same as what would result from a Taylor series expansion of the true policy function $\alpha_{t}$, around the deterministic steady state. Following Schmitt-Grohé and Uribe (2004) and Jin and Judd (2002) we can think of the true policy function in a recursive economy as a function that depends on the model's state variables, $x_{t}$, and on a parameter that scales the variancecovariance matrix of the model's exogenous shock processes, $\sigma$; that is, $\alpha_{t}=\alpha\left(x_{t}, \sigma\right)$. A Taylor series to policy function $\alpha_{t}$, evaluated at approximation points $x_{t}=\bar{x}$ and $\sigma=0$, would then result in:

$\alpha_{t}=\alpha(\bar{x}, 0)+\alpha_{x}(\bar{x}, 0) \widehat{x}_{t}+\alpha_{\sigma}(\bar{x}, 0) \sigma+\frac{1}{2} \widehat{x}_{t}^{\prime} \alpha_{x x}(\bar{x}, 0) \widehat{x}_{t}+\alpha_{x \sigma}(\bar{x}, 0) \widehat{x}_{t} \sigma+\frac{1}{2} \alpha_{\sigma \sigma}(\bar{x}, 0) \sigma^{2}+\ldots$

That is, in contrast to the Taylor series expansion in equation (18) the DS approximate portfolio solution does only consider how variations in the model's state variables affect the optimal portfolio solution, but ignores the effect of variations in the size of uncertainty. ${ }^{15}$ In

\footnotetext{
${ }^{14}$ Because $W_{h t}$ can be expressed as a function of $\omega_{t}$.

${ }^{15}$ The comparison of the DS solution with equation (18) is simply for reasons of exposition. We are of
} 
the general case of a dynamic model as in the present setup, this still does not imply that the size of uncertainty cannot have an effect on optimal portfolios. In particular, there could be an effect of the size of uncertainty, $\sigma$, on the portfolio through the effect of $\sigma$ on the states, $\widehat{x}_{t}$, themselves. In order for this to happen, one needs to approximate the macroeconomic model at least to order two (or higher): Schmitt-Grohé and Uribe (2004) show that the perturbation solution for model variables are not affected by $\sigma$ at first-order (certainty equivalence) and only through a constant at second-order. In the present paper, whenever we present results from the local solution method, this means that we use the DS method to characterize firstorder accurate portfolio dynamics in conjunction with second-order accurate dynamics for all other variables, making use of the solution files provided by Schmitt-Grohé and Uribe (2004). Section 5.2 studies the consequences of the negligence of a (direct) dependence of the portfolio solution on the scale of uncertainty under the DS method.

We now briefly mention another problem that is not explicitly addressed in the description of the DS solution method. The problem arises because, in general, in incomplete market open economy models the deterministic steady-state NFA positions cannot be determined uniquely, which is of particular relevance in asymmetric country settings where the stationary distribution of net foreign assets is likely not to be centered around zero. While introducing a stationarity inducing-device such as an endogenous discount factor (as we have in our test model) allows to obtain a unique $\overline{W_{h}}$ at the deterministic steady state, this obtained value may be very different from its stochastic or risky steady state value. Coeurdacier et al. (2011) define the risky steady state to be the 'point where agents choose to stay at a given date if they expect future risk and if the realization of shocks is 0 at this point'. In section 4.2.2 we explore alternatives to the (exogenously) pinned down deterministic steady state value of $\overline{W_{h}}$ as possible better approximation points, and evaluate the performance of the DS method in these cases. We consider using the mean of the ergodic NFA distribution from the global method, or using the stochastic steady state value found by an iterative algorithm proposed by Devereux and Sutherland (2009) as alternatives. We also discuss the relation to the 'risky steady state' literature (see, Coeurdacier et al. (2011), Julliard (2011), De Groot (2013)).

Finally, we would like to emphasize another technical difficulty with the perturbation method. It arises when simulations are generated using second or higher order approximation to the model equilibrium system. In this case the dynamics of control variables are affected by higher than second order terms. These in turn feed into dynamics of the state. This can lead to explosive system dynamics because, as emphasized by Kim et al. (2003), these extra high-order terms in general do not correspond to high-order coefficients in a Taylor series approximation. A 'stable simulation' can be obtained by 'pruning' out extraneous high-order terms in each iteration by computing projections of second-order terms based on a first-order approximation. Our simulations obtained using the perturbation solution use 'pruning'. Yet, the latter lacks theoretical justification and so it is merely a trick. See Kim et al. (2003), Den Haan and de Wind (2009), Lombardo (2010) and Lan and Meyer-Gohde (2013) for a discussion of advantages and disadvantages of 'pruning'.

course not suggesting that an approximate solution to the true unknown portfolio function actually can be obtained by taking a simple Taylor series expansion around the non-stochastic steady state. This is not feasible using standard local approximation methods (using the standard implicit function theorem) - the portfolio is indeterminate both at the non-stochastic steady state and in a first-order approximation of the stochastic setting. This is exactly the problem that the DS method (and Judd and Guu (2001) in their bifurcation approach) have addressed and proposed (different) ways of solving for. 


\section{Model specification 1: two equities}

We start our comparison of the two solution methods by looking at a scenario in which the bond market of the model framework presented in section 2 is shut down by a zero-borrowing limit in the bond for both countries, i.e. $b_{t}^{a} \geq 0$, for $a=h, f$. In this case, our model framework becomes equivalent to the workhorse model of Devereux and Sutherland (2011). The budget constraint of the representative agent in country $a$ becomes:

$$
c_{a t}+\theta_{h t}^{a} q_{h t}+\theta_{f t}^{a} q_{f t}=\theta_{h t-1}^{a}\left(q_{h t}+Y_{h t}^{k}\right)+\theta_{f t-1}^{a}\left(q_{f t}+Y_{f t}^{k}\right)+Y_{a t}^{l} .
$$

We first (section 4.1) consider a setting where the two countries of our model economy are parameterized in an entirely symmetric way. This facilitates the comparison because in this case a net foreign asset position of zero $\left(\overline{W_{h}}=0\right)$ is the 'natural' approximation point for the local method. ${ }^{16}$ This is equivalent to both countries holding equal wealth shares: $\bar{\omega}=0.5$. Such setting can be thought of as a relevant description of financial trade between similar countries or country groups, e.g. financial trade between advanced economies. Section 4.2 repeats the analysis for an asymmetric setup. The particular asymmetry we consider here is that we subject the foreign country to twice as volatile income endowment shocks. This captures one particular dimension in which emerging market economies are different from advanced economies - they are subject to substantially higher macroeconomic volatility at business cycle frequencies, see e.g. Aguiar and Gopinath (2007). ${ }^{17}$ The asymmetric setup of model specification 1 is also relevant from a numerical point of view. In particular, it allows us to clarify how the performance of the local (DS) solution is affected by the choice of the approximation point, which we discuss in section 4.2.2.

We compare global and DS solution methods by contrasting policy functions, model moments of simulated time series over both short and long horizons, and welfare measures.

\subsection{Symmetric setting}

\begin{tabular}{llr} 
Parameter & & Value \\
\hline Discount factor & $\beta$ & 0.95 \\
Endogenous discount factor & $\eta$ & 0.001 \\
Risk aversion & $\gamma_{h}, \gamma_{f}, 1 / \psi_{h}, 1 / \psi_{f}$ & 2.00 \\
Capital income share & $Y_{h}^{k} / Y_{h}, Y_{f}^{k} / Y_{f}$ & 0.30 \\
Annual mean output & $\bar{Y}_{h}, \bar{Y}_{f}$ & 1 \\
Persistence & $\rho_{Y_{h}^{k}}, \rho_{Y_{f}^{k}}, \rho_{Y_{h}^{l}}, \rho_{Y_{f}^{l}}$ & 0.80 \\
Volatility & $\sigma_{Y_{h}^{k}}, \sigma_{Y_{f}^{k}}, \sigma_{Y_{h}^{l}}, \sigma_{Y_{f}^{l}}$ & 0.02 \\
Correlation & $c o r\left(Y_{h}^{k}, Y_{h}^{l}\right)=\operatorname{cor}\left(Y_{f}^{k}, Y_{f}^{l}\right)$ & 0.20 \\
borrowing constraint & $B L$ & 0
\end{tabular}

Table 2: Parameters for the symmetric setup, model specification 1.

The parameter values for the setup with symmetric countries are reported in table 2 . They fall into the range of values that are commonly used in macroeconomics. In particular, the discount factor $\beta$ of 0.95 implies an annual interest rate of about $5 \%$. We set the inverse

\footnotetext{
${ }^{16}$ As explained in detail later (section 4.2.2) the functional form of our endogenous discount factor implies that $\overline{W_{h}}=0$ at the deterministic steady state.

${ }^{17}$ Section 5 will deal with other, more substantial asymmetries between advanced and emerging economies.
} 

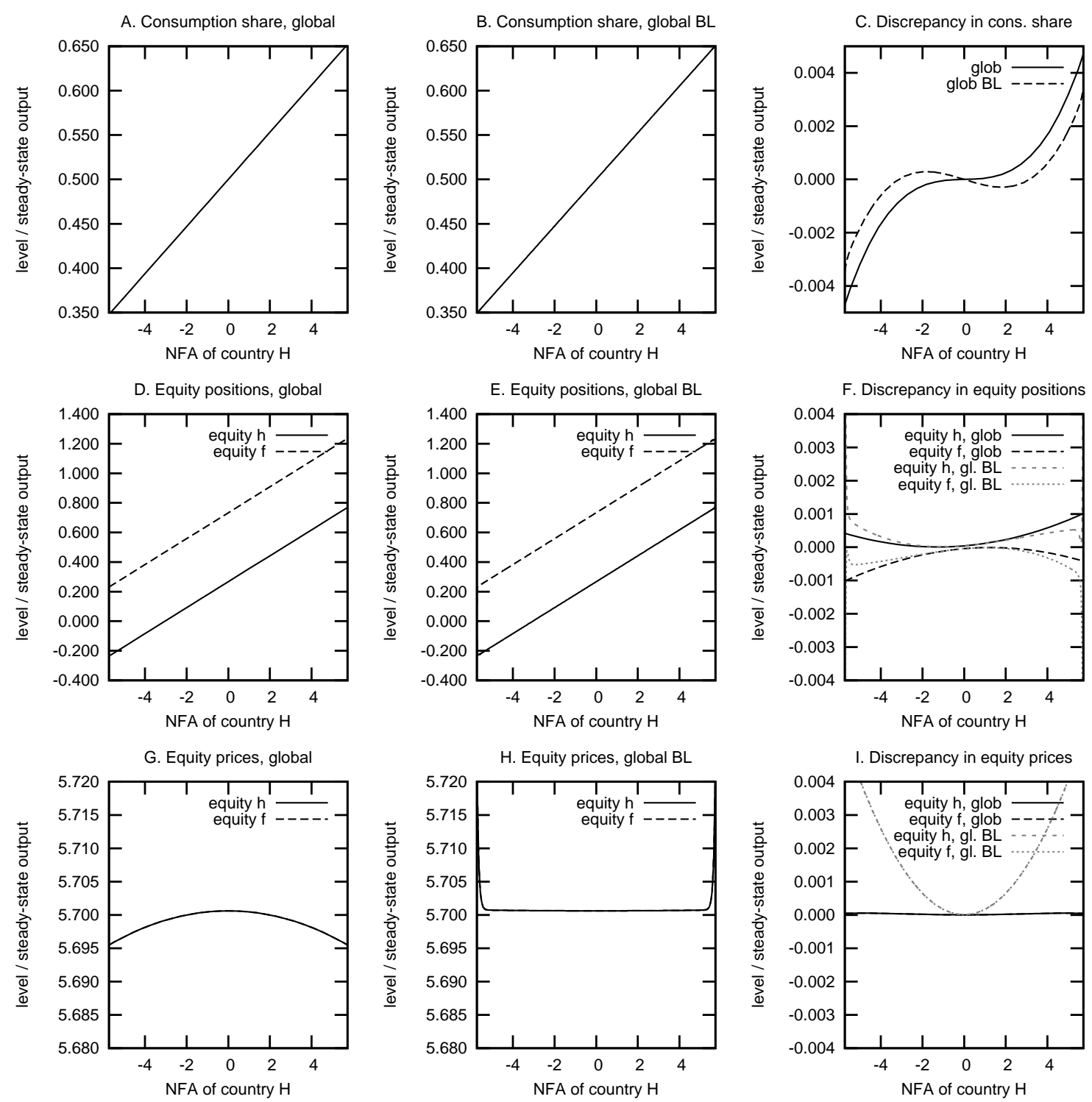

Figure 1: Country h's policy functions in the symmetric setting, model specification 1. Panels A,D,G present the policy functions for the global solution method with EDF. Panels B,E,H do the same for the global solution with borrowing limits. Panels C,F,I plot the discrepancy between the perturbation and the respective global policy functions.

elasticity of intertemporal substitution equal to the coefficient of relative risk aversion, i.e. $\gamma_{a}=1 / \psi_{a}$ for $a=h, f$, which implies CRRA preferences. The coefficients of relative risk aversion, $\gamma_{h}$ and $\gamma_{f}$, are set to 2 , a commonly chosen value. The means of the total endowment incomes are normalized to 1 , of which capital income accounts for $30 \%$. The persistences of the income endowment processes, $\rho_{Y_{a}^{k}}, \rho_{Y_{a}^{l}}$, for $a=h, f$, are set to 0.8 , their volatilities, $\sigma_{Y_{a}^{k}}$, $\sigma_{Y_{a}^{l}}$, for $a=h, f$, to $0.02 .{ }^{18}$ These choice imply a standard deviation of aggregate log-output

\footnotetext{
${ }^{18}$ That is, table 2 reports the parameters of the variance-covariance matrix for outputs, $\Sigma_{\mathbf{Y}}$. Given this, we
} 
of $\sigma\left(\log Y_{h}\right)=\sigma\left(\log Y_{f}\right)=0.0163$, roughly in line with the values from an estimated $\mathrm{AR}(1)$ process of postwar US annual output. We assume a positive correlation between countries' labor and capital income, equal to 0.2 . The moments of the output processes, $\mathbf{Y}_{t}$, that we use in all the numerical results that follow, are the 'targets' that we use to create discrete approximations to continuous VAR processes. Because discrete approximations are not exact, we use numerically computed moments as inputs to the DS method. This avoids differences across the two methods to arise from the discretization of the exogenous processes. Finally, we treat the endogenous discount factor as a purely technical device that induces stationarity and throughout the paper set $\eta=10^{-3}$, a 'small' value. For solving the model with the global method plus borrowing constraint, we set $B L=0$ (and $\eta=0$ ). For our choice of specification of a 'joint constraint' and parameter $B L=0$, the constraints become binding less than half a percent of the times in a stochastic simulation, and allows the NFA position to reach about the sixfold of annual steady state output. ${ }^{19}$

The left column (panels A,D,G) in figure 1 presents policy functions for the home country's consumption share, portfolio shares and asset prices for the global solution method. We plot policies as a function of the home country's NFA and conditional on $\mathbf{Y}=E[\mathbf{Y}]$. The solution is highly accurate as evidenced by the Euler equation errors presented in figure 11 in appendix A. Because of its high accuracy we refer to the global solution as to the true solution of the model. The middle column (panels B, E, H) repeats the policy functions for the global solution method with borrowing limits.

The differences between the perturbation and global methods' policy functions are plotted in the right column (panels C,F,I). First, local and global solutions predict slightly different consumption shares for country $h$ when country $h$ 's NFA is far from 0 . The relative difference can be as large as 0.005. But the levels of NFA where the difference is large are unlikely. The shape of asset price policies is influenced a lot by the presence of the EDF. In the global solution without EDF (but with borrowing limits) asset prices increase when one of the countries becomes significantly richer than the other. The discrepancy in asset prices between local and global solution with EDF is negligible.

We next turn to contrast results from global and DS solutions based on simulated model data. Here we consider simulations over both short and long horizons, to be able to evaluate the methods' performance both at short horizons and in terms of ergodic model properties. Figure 2 compares time paths generated using the perturbation and global solution methods, obtained from one particular realization of exogenous variables for 100 periods. Simulations for the perturbation solution are based on a second-order approximation and were 'pruned'. Except for portfolio holdings, the two solution methods with EDF generate similar simulation paths: the maximum difference for the NFA, consumption share, and the asset prices are respectively $0.36 \%, 0.002 \%$, and $0.0003 \%$. The maximum difference between the simulated series of portfolio holdings is $2.67 \%$. The 'portfolio errors' are virtually perfectly negatively correlated: $\rho\left(\theta_{h}^{h, D S}-\theta_{h}^{h, g l o b}, \theta_{f}^{h, D S}-\theta_{f}^{h, g l o b}\right)=-1$. So, despite a large discrepancy in simulated

can compute the variance-covariance matrix of disturbances, $\Sigma_{\varepsilon}$, as $\Sigma_{\varepsilon}=\Sigma_{\mathbf{Y}}-\Lambda \Sigma_{\mathbf{Y}} \Lambda^{\prime}$, where $\Lambda$ is the matrix of autocorrelations, containing the persistences of the diagonal.

${ }^{19}$ The size of $\eta$ is mostly consequential for the shape of ergodic distributions. For the short time paths studied so far, changing $\eta$ (within reasonable bounds) had little impact on the results. In section 4.2 we demonstrate how the ergodic distributions obtained using both local and global solution method depends on the choice of $\eta$. We show that, in asymmetric country settings, it affects not only standard deviation of ergodic distributions but also the mean. We similarly demonstrate, in appendix B.2, the effect of the tightness of the borrowing constraint, $B L$, when the model is solved with the global method plus borrowing constraint. 

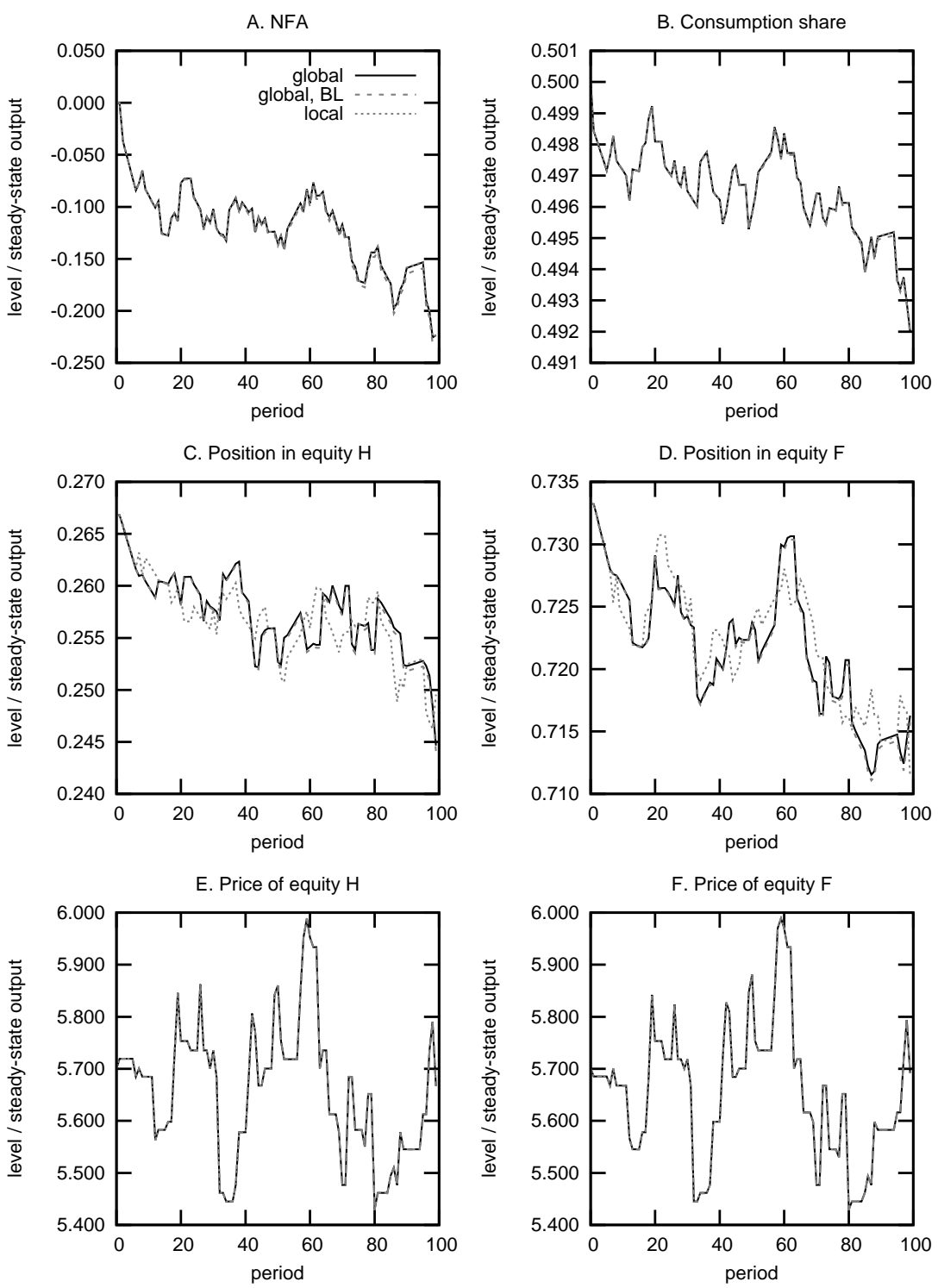

Figure 2: Simulated time paths for country $h$ in the symmetric setting, model specification 1 .

Labels 'global' and 'local' refer, respectively, to the global and DS solutions when the endogenous discount factor is present. Label 'global $\mathrm{BL}$ ' refers to the global solution when a borrowing limit is present.

portfolios the two NFA paths are close.

Next we compare first- and second-order moments obtained using the two solution methods. Table 3 reports moments from both a 'panel simulation' (first subtable) and from a long simulation (second subtable, labeled 'ergodic moments'). By 'panel simulation' we refer to a simulation of 10000 series of 100 periods, starting at $W_{h 0}=0$, each. The long simulation instead is a single series of 100 million periods. Because the NFA position is highly persistent, moments obtained from a panel of short simulations and ergodic moments are generally different. Looking at the moments from panel simulations, the local and global solution methods generate identical means and standard deviations. Yet, the solutions differ in their 
predictions for correlations of portfolios with H's and F's output. While the perturbation solution predicts the correlation signs correctly, it underestimates the strength of the relation. For example, it predicts that country $h$ 's ownership of asset $\mathrm{H}$ is nearly uncorrelated with output in the two countries. The global solution method implies a relation of mild strength: $\rho\left(\theta_{h}^{h}, Y^{h}\right)=-0.194, \rho\left(\theta_{f}^{h}, Y^{h}\right)=0.153$. Correlations implied by the perturbation solution are smaller because perturbation solution method imposes that 'to a first-order approximation, the portfolio excess returns are zero-mean i.i.d. random variables. ${ }^{20}$ The excess returns drive the portfolio choice and render it largely unrelated to the fundamentals.

Looking at ergodic moments, the local solution gives almost identical results as the global when compared to the version that incorporates the EDF (column 'global'). The ergodic moments from the global solution with borrowing limits (column 'global BL') show the same ergodic means, but a much larger standard deviation, especially for the NFA position. This is visualized in figure 3, which plots the ergodic distribution of NFA for three cases of table 3. (Note that the stationary distribution of 'local' and 'global' virtually coincide.)

Finally, we also compute welfare differences across the two methods: $\Delta(\%)$ measures the percent difference in certainty equivalent consumption of the local method compared to the global method, conditional on $\mathbf{Y}=E[\mathbf{Y}]$ and $W_{h 0}=0 .{ }^{21}$ Measured in terms of certainty equivalent consumption (of both home and foreign agent), welfare is found to be $0.0158 \%$ higher under the local method than under 'global', and $0.0160 \%$ higher than under 'global BL'.22

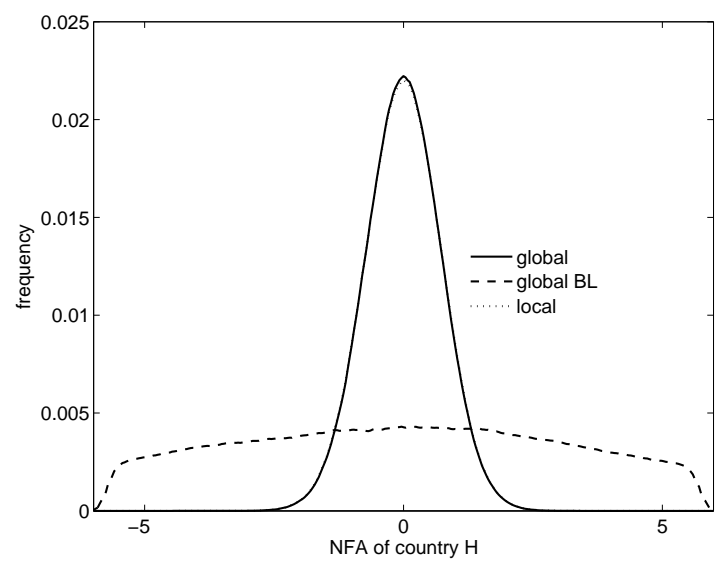

Figure 3: Ergodic distribution of NFA holdings in the symmetric setting, model specification 1 .

Labels 'global' and 'local' refer, respectively, to the global and DS solutions when the endogenous discount factor is present. Label 'global $\mathrm{BL}$ ' refers to the global solution when a borrowing limit is present.

To summarize, in a symmetric setting parameterized to match output processes of developed economies the perturbation method performs well. In particular, it matches closely

\footnotetext{
${ }^{20}$ For details see page 1329 in Devereux and Sutherland (2010).

${ }^{21}$ In particular, our welfare measure is defined as $\Delta(\%)=\left(\frac{\text { welfare }_{a}^{D S}\left(c_{a}^{C E}\right)}{\text { welfare }_{a}^{\text {glob }}\left(c_{a}^{C E}\right)}-1\right) \times 100$, where $a=h, f$.

${ }^{22}$ Despite the higher variance of net foreign assets in the case of 'global BL' compared to 'global' the welfare differences under these scenarios with respect to 'local' are close. We explain this as the realizations of the time paths of consumptions of 'global BL' and 'global' that are relevant for the welfare measures are similar, and consumption time paths become different only at large $t$, in which case they are heavily discounted.
} 
Moments from panel simulations

\begin{tabular}{crrr|rrr||rrr} 
& \multicolumn{3}{c|}{ global } & \multicolumn{3}{c||}{ global BL } & \multicolumn{3}{c}{ local } \\
& $\mu()$. & $\sigma()$. & $\rho\left(., Y^{h}\right)$ & $\mu()$. & $\sigma()$. & $\rho\left(., Y^{h}\right)$ & $\mu()$. & $\sigma()$. & $\rho\left(., Y^{h}\right)$ \\
\hline$N F A^{h}$ & 0.001 & 0.081 & -0.119 & 0.001 & 0.083 & -0.097 & 0.001 & 0.081 & -0.095 \\
$c_{h}$ & 0.500 & 0.002 & 0.251 & 0.500 & 0.002 & 0.247 & 0.500 & 0.002 & 0.251 \\
$\theta_{h}^{h}$ & 0.267 & 0.007 & -0.194 & 0.267 & 0.007 & -0.195 & 0.267 & 0.007 & -0.070 \\
$\theta_{f}^{h}$ & 0.733 & 0.007 & 0.153 & 0.733 & 0.007 & 0.149 & 0.733 & 0.007 & 0.028 \\
$q_{h}$ & 5.703 & 0.111 & 0.737 & 5.703 & 0.111 & 0.737 & 5.704 & 0.111 & 0.737 \\
$q_{f}$ & 5.703 & 0.111 & 0.648 & 5.703 & 0.111 & 0.648 & 5.704 & 0.111 & 0.648 \\
$r_{h}$ & 1.053 & 0.014 & 0.243 & 1.053 & 0.014 & 0.242 & 1.053 & 0.014 & 0.243 \\
$r_{f}$ & 1.053 & 0.014 & 0.180 & 1.053 & 0.014 & 0.180 & 1.053 & 0.014 & 0.180
\end{tabular}

Ergodic moments

\begin{tabular}{|c|c|c|c|c|c|c|}
\hline & & $\begin{array}{c}\text { global } \\
\sigma(.)\end{array}$ & $\mu()$. & $\begin{array}{c}\text { global BL } \\
\sigma(.)\end{array}$ & $\mu()$. & $\begin{array}{l}\text { local } \\
\sigma(.)\end{array}$ \\
\hline$N F A^{h}$ & -0.004 & 0.722 & -0.006 & 3.041 & -0.005 & 0.727 \\
\hline$c_{h}$ & 0.500 & 0.019 & 0.500 & 0.080 & 0.500 & 0.019 \\
\hline$\theta_{h}^{h}$ & 0.267 & 0.063 & 0.267 & 0.267 & 0.267 & 0.064 \\
\hline$\theta_{f}^{h}$ & 0.733 & 0.063 & 0.732 & 0.267 & 0.733 & 0.064 \\
\hline$q_{h}$ & 5.703 & 0.118 & 5.704 & 0.118 & 5.703 & 0.118 \\
\hline$q_{f}$ & 5.703 & 0.118 & 5.704 & 0.118 & 5.703 & 0.118 \\
\hline$r_{h}$ & 1.053 & 0.014 & 1.053 & 0.014 & 1.053 & 0.014 \\
\hline$r_{f}$ & 1.053 & 0.014 & 1.053 & 0.014 & 1.053 & 0.014 \\
\hline
\end{tabular}

Table 3: Comparison of model moments, symmetric setting of model specification 1.

Columns 'global' and 'local' refer, respectively, to the global and DS solutions when the endogenous discount factor is present. Column 'global BL' refers to the global solution when a borrowing limit is present.

the evolution of macroeconomic variables and of the NFA position. It produces somewhat inaccurate predictions about cyclical properties of countries' portfolios. These findings are also robust with respect to increasing shock volatility, increasing shock persistence or higher risk aversion.

\section{$4.2 \quad$ Asymmetric setting}

In this section we study a setting in which country $f$ faces income shocks with higher volatility, serving as a stylized example of financial trade between advanced economies and emerging market economies. In particular, we assume $\sigma_{Y_{f}^{i}}=2 \sigma_{Y_{h}^{i}}$, for $i=k, l$. The implied standard deviations of aggregate $\log$-outputs in this case are $\sigma\left(\log Y_{h}\right)=0.0163$ and $\sigma\left(\log Y_{f}\right)=0.0324$. Because markets are incomplete, precautionary motives are active. Since shocks that country $f$ faces are more volatile, its precautionary demand is higher. So, we expect country $f$ (country $h$ ) to accumulate more (less) wealth on average, and thus expect the stationary distribution of NFA to be no longer centered around zero, but around a negative value. This setting is interesting from a methodological point of view, because the perturbation solution method, being a local method, requires a 'point' around which the approximation is taken, often chosen to be the deterministic steady state of the economic model. However, in the deterministic version of the model the two countries remain symmetric - asymmetries in our example were only specified in shock volatilities; in the limit where those shock volatilities go to zero, the two economies become symmetric again. This instructs us to continue to approximate the 
model around a NFA position of $\overline{W_{h}}=0 .{ }^{23}$ This case presents us with a realistic setting where we can expect the perturbation solution quality to deteriorate as the simulated NFA position deviates from this approximation point. At the same time the solution accuracy of the global solution method should not be compromised. This is indeed true as measured by the errors in the equilibrium conditions plotted in figure 12, in appendix A.
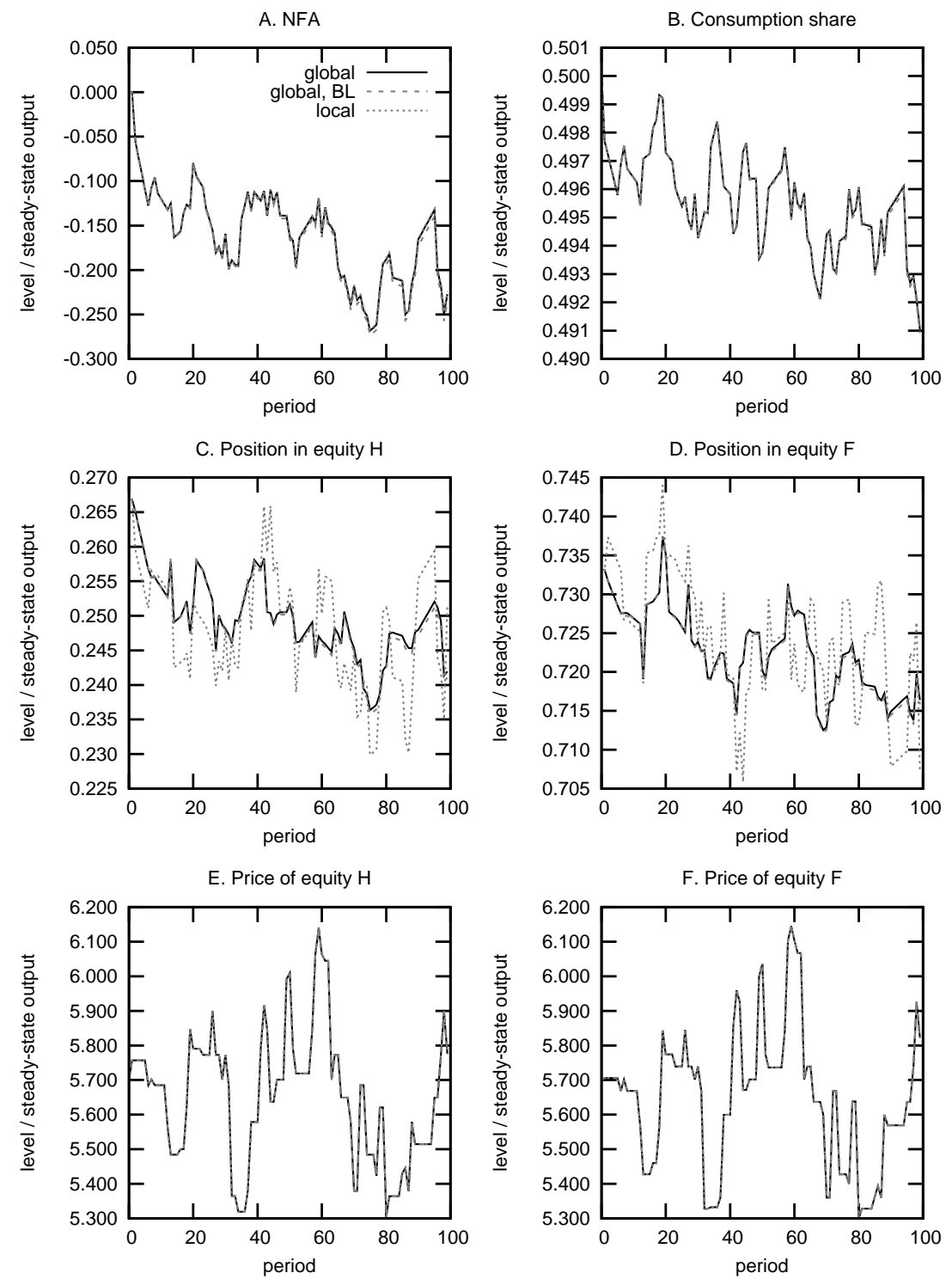

Figure 4: Simulated time paths for country $h$ in the asymmetric setting with $\sigma_{Y_{f}^{i}}=2 \sigma_{Y_{h}^{i}}$, for $i=k, l$, model specification 1 .

Labels 'global' and 'local' refer, respectively, to the global and DS solutions when the endogenous discount factor is present. Label 'global $\mathrm{BL}$ ' refers to the global solution when a borrowing limit is present.

Figure 4 plots simulated series for the setting with diverse output volatility. Results are qualitatively similar to those for the symmetric setting (see figure 2). Consumption, asset

\footnotetext{
${ }^{23}$ This, in fact, is the deterministic steady state value of the NFA implied by the presence of our assumption of an endogenous discount factor, which insures this deterministic steady state is also well defined.
} 
prices and NFA are approximated well. But portfolio dynamics differ across the two solution methods. The maximal error for the NFA is $1.20 \%$ of country $h$ 's output, for the consumption share and asset prices it is 0.011 and $0.0015 \%$ respectively. The maximal error for portfolios is, with $6.20 \%$, more substantial. The first part of table 4 shows moments from 'panel simulations', computed from 10000 randomly generated samples of length 100, each starting at $W_{h 0}=0$. The perturbation solution method produces almost identical results for means and standard deviations as the global solutions; it predicts cyclical properties of the portfolio slightly incorrectly. For example, $\rho\left(\theta_{f}^{h}, Y^{f}\right)=0.223$ in the perturbation solution while it is 0.353 in the global solution (0.349 in the global solution with borrowing limits). Another result becomes apparent from the subtable on 'panel simulations': for moments of short time series, the model versions with EDF (column 'global') or without EDF but borrowing limits (column 'global BL') deliver equivalent results. Overall, the local (DS) solution performs well at short horizons, despite the potential difficulties steming from approximating at the (symmetric) deterministic steady state.

Moments from panel simulations

\begin{tabular}{crrr|rrr||rrr} 
& \multicolumn{3}{c|}{ global } & \multicolumn{3}{c||}{ global BL } & \multicolumn{3}{c}{ local } \\
& $\mu()$. & $\sigma()$. & $\rho\left(., Y^{h}\right)$ & $\mu()$. & $\sigma()$. & $\rho\left(., Y^{h}\right)$ & $\mu()$. & $\sigma()$. & $\rho\left(., Y^{h}\right)$ \\
\hline$N F A^{h}$ & -0.004 & 0.129 & -0.060 & -0.004 & 0.131 & -0.061 & -0.004 & 0.129 & -0.059 \\
$c_{h}$ & 0.500 & 0.004 & 0.158 & 0.500 & 0.004 & 0.155 & 0.500 & 0.004 & 0.158 \\
$\theta_{h}^{h}$ & 0.267 & 0.015 & -0.235 & 0.267 & 0.016 & -0.235 & 0.267 & 0.017 & -0.176 \\
$\theta_{f}^{h}$ & 0.733 & 0.009 & 0.353 & 0.733 & 0.009 & 0.349 & 0.732 & 0.012 & 0.223 \\
$q_{h}$ & 5.708 & 0.168 & 0.482 & 5.708 & 0.169 & 0.482 & 5.708 & 0.168 & 0.482 \\
$q_{f}$ & 5.709 & 0.183 & 0.389 & 5.709 & 0.184 & 0.389 & 5.709 & 0.183 & 0.389 \\
$r_{h}$ & 1.053 & 0.020 & 0.162 & 1.053 & 0.020 & 0.162 & 1.053 & 0.020 & 0.162 \\
$r_{f}$ & 1.053 & 0.023 & 0.109 & 1.053 & 0.023 & 0.109 & 1.053 & 0.023 & 0.109
\end{tabular}

Ergodic moments

\begin{tabular}{|c|c|c|c|c|c|c|}
\hline & & global & & lobal BL & & local \\
\hline$N F A^{h}$ & -0.168 & 1.111 & -1.661 & 2.872 & -0.179 & 1.152 \\
\hline$c_{h}$ & 0.495 & 0.030 & 0.456 & 0.076 & 0.495 & 0.030 \\
\hline$\theta_{h}^{h}$ & 0.248 & 0.130 & 0.074 & 0.334 & 0.247 & 0.135 \\
\hline$\theta_{f}^{h}$ & 0.723 & 0.066 & 0.635 & 0.171 & 0.722 & 0.068 \\
\hline$q_{h}$ & 5.708 & 0.179 & 5.709 & 0.180 & 5.708 & 0.178 \\
\hline$q_{f}$ & 5.709 & 0.194 & 5.710 & 0.195 & 5.709 & 0.194 \\
\hline$r_{h}$ & 1.053 & 0.020 & 1.053 & 0.021 & 1.053 & 0.020 \\
\hline$r_{f}$ & 1.053 & 0.022 & 1.053 & 0.022 & 1.053 & 0.022 \\
\hline
\end{tabular}

Table 4: Comparison of model moments in the asymmetric setting with $\sigma^{f}=2 \sigma^{h}$, model specification 1 .

Columns 'global' and 'local' refer, respectively, to the global and DS solutions when the endogenous discount factor is present. Column 'global BL' refers to the global solution when a borrowing limit is present.

The second subtable of table 4 summarizes the findings on ergodic moments in the asymmetric country scenario. The perturbation method produces ergodic moments that are very close to the global method, when both methods are applied to the model with an EDF (columns 'global' and 'local'), yet it produces markedly different moments when compared to the global solution method without EDF but borrowing limits instead ('global BL'). ${ }^{24}$ From

\footnotetext{
${ }^{24}$ De Groot et al. (2014) also compare global and local solution methods of incomplete market general equilibrium models, but without portfolio choice. They compare a model with borrowing constraints (solved globally) and a model with a debt-elastic interest rate (solved locally). Because their interest lies mainly in the ergodic moments they find significant differences between the global and local solution method. This mirrors
} 
this we learn two lessons. One, the EDF has a very strong impact on the ergodic properties of the model, influencing not only the volatilities of variables in a stochastic simulation (as can be expected) but also strongly affecting their means. In particular, an EDF with our parameter choice of $\eta=10^{-3}$ leads the stationary distribution of NFA to be only slightly asymmetric, as witnessed by an ergodic mean of the NFA of -0.168 ('global'), instead of -2.55 in the model version without EDF ('global BL'). If we set $\eta=10^{-2}$, this mean shifts to -0.022 , and is almost entirely symmetric. This means that the EDF dominates any other, economic forces that may play a role in determining asymmetries in the ergodic distribution, and thus that the need or convention to resort to stationarity-inducing devices when using local solution methods such as the DS approach precludes an economic interpretation of longrun model moments. While this should not be interpreted as a 'failure' of the DS method itself - which, comparing the columns labeled 'global' and 'DS' continues to work accurately - this comes as an unwanted by-product from the convention of using a stationarity-inducing device when solving incomplete market models with local approximation methods.

To provide a deeper understanding of the role of the EDF in shaping ergodic moments, figure 5 plots the ergodic distribution of NFA for the three cases of table 4 (global and local solution with $\mathrm{EDF}$ and $\eta=10^{-3}$, and 'global BL'), together with ergodic distributions for several parameter values of $\eta$. For a value of $\eta=10^{-4}$ the ergodic distributions reflect much more the underlying economic asymmetries of our parameterization, the means of the NFA distributions in this case $\left(-1.397\right.$ for 'global $\eta=10^{-4}$, and -1.740 for 'local $\eta=$ $10^{-4}$ ') are much closer to the model without EDF ('global BL'). However, it is also the case that the differences between local and global solutions become more pronounced in this case, ergodic mean consumption shares, domestic holdings of home and foreign equity are 0.456, $0.064,0.6311$ for the 'global $\eta=10^{-4}$, , but $0.463,0.163,0.652$ for 'local $\eta=10^{-4}$ '. For a value of $\eta$ of $10^{-5}$, the influence of the EDF on the ergodic distribution starts to vanish: the ergodic distribution of 'global $\eta=10^{-5}$ ' is close to the one it converges to when $\eta=0$, while the ergodic distribution of NFA when solved with the local method no longer produces a reasonable distribution of NFA holdings, it produces a very flat, near nonstationary distribution with a mean of -17.711 .

Welfare differences are somewhat larger than in the symmtric country setup. Welfare, $\Delta(\%)$, is found to be $0.0237 \%$ and $0.0556 \%$ higher under the local method compared to'global', when considering certainty equivalent consumption of the home agent or the foreign agent respectively; and it is $0.0240 \%$ and $0.0560 \%$ higher than under 'global BL'.

To summarize, the DS perturbation solution in an asymmetric country setting of model 1 remains accurate along short time paths and moments from 'panel simulations'. When characterizing ergodic moments, the DS method faces a technical difficulty that comes from the (need to) use of stationarity-inducing devices. When the forces of the EDF are strong, the asymmetries coming from structural differences in the two economies are largely downplayed. Since the ergodic distributions remain surprisingly symmetric in our baseline parameterization the global and local (with EDF parameter $\eta=10^{-3}$ ) issues of taking the local approximation around the 'right' approximation point are of minor importance. The focus of the next section is to evaluate the generality of this finding, and to present a sensitivity analysis of the asymmetric country setting.

our results on ergodic model properties. 


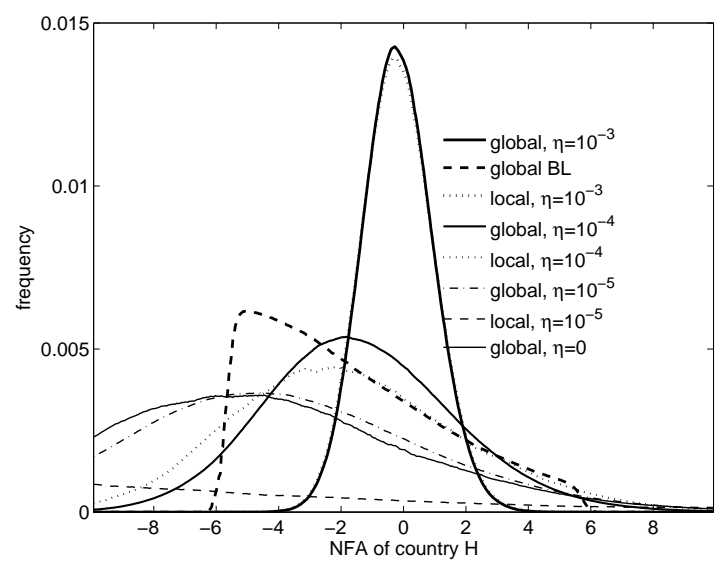

Figure 5: Ergodic distribution of NFA holdings in the asymmetric setting with $\sigma_{Y_{f}^{i}}=2 \sigma_{Y_{h}^{i}}$, for $i=k, l$, model specification 1 .

Labels 'global' and 'local' refer, respectively, to the global and DS solutions when the endogenous discount factor is present. Label 'global $\mathrm{BL}$ ' refers to the global solution when a borrowing limit is present.

\subsubsection{Sensitivity analysis in the asymmetric setting}

The particular sensitivity experiment we present is that of increasing the volatilities of country $h$ and $f$ to $\sigma_{Y_{h}^{k}}=\sigma_{Y_{h}^{l}}=0.03$ and $\sigma_{Y_{f}^{k}}=\sigma_{Y_{f}^{l}}=0.06$, and of modifying our parameter of risk aversion from the benchmark value of 2 to a substantially higher value of $15 .^{25}$

Table 5 summarizes simulated model moments, from both 'panel simulations' and ergodic moments. The model moments from 'panel simulations' document that at short horizons, even for this more extreme parameterization, the local method continues to capture the behavior of variables accurately. We thus focus on ergodic model properties. Here, larger differences between the two solutions emerge. As risk-aversion increases, precautionary demands of both countries increase. But country $f$, facing more volatile shocks, increases its demand more. The economic forces of asymmetries are now magnified (and work more strongly against the symmetry-inducing influence of the EDF parameter $\eta=10^{-3}$ ), leading to a) strong asymmetries in ergodic distributions of our model variables, and b) to much more substantial differences in local and gobal solution methods - potentially because the 'point of approximation' of the deterministic steady state is a less suitable description of the stochastic economy.

Figure 6 repeats the figure on ergodic distributions of NFA holdings for our sensitivity experiment. It shows ergodic distributions of NFA for the baseline parameter value of $\eta=$ $10^{-3}$ (which corresponds to the simulation results in table 5), and also reports distributions for a lower value of $\eta=10^{-4}$. For the global solution already $\eta=10^{-4}$ is a low enough value not to assert too strong an influence on the shape of the ergodic distribution and is close to the distributions implied by 'global $\eta=10^{-5}$ ' or 'global $\eta=0$ '. On the other hand, at $\eta=10^{-4}$ the local method no longer produces a distribution within reasonable ranges of NFA holdings, its mean being at -40.697 .

\footnotetext{
${ }^{25}$ Note that the implied standard deviation of aggregate log-output is more moderate, i.e. $\sigma\left(\log Y_{h}\right)=0.0244$ and $\sigma\left(\log Y_{f}\right)=0.0486$. Also, because of Epstein-Zin preferences the elasticity of intertemporal substitution remains at its reasonable value of $1 / 2$.
} 
Moments from panel simulations

\begin{tabular}{|c|c|c|c|c|c|c|c|c|c|}
\hline & \multicolumn{3}{|c|}{ global } & \multicolumn{3}{|c|}{ global BL } & \multicolumn{3}{|c|}{ local } \\
\hline & $\mu()$. & $\sigma()$. & $\rho\left(., Y^{h}\right)$ & $\mu()$. & $\sigma()$. & $\rho\left(., Y^{h}\right)$ & $\mu()$. & $\sigma()$. & $\rho\left(., Y^{h}\right)$ \\
\hline$N F A^{h}$ & -0.111 & 0.198 & -0.065 & -0.113 & 0.203 & -0.066 & -0.113 & 0.200 & -0.055 \\
\hline$c_{h}$ & 0.498 & 0.006 & 0.156 & 0.498 & 0.006 & 0.154 & 0.498 & 0.006 & 0.154 \\
\hline$\theta_{h}^{h}$ & 0.259 & 0.032 & -0.210 & 0.261 & 0.027 & -0.211 & 0.261 & 0.027 & -0.164 \\
\hline$\theta_{f}^{h}$ & 0.724 & 0.024 & 0.251 & 0.721 & 0.018 & 0.250 & 0.721 & 0.018 & 0.228 \\
\hline$q_{h}$ & 5.757 & 0.255 & 0.481 & 5.757 & 0.255 & 0.481 & 5.758 & 0.255 & 0.482 \\
\hline$q_{f}$ & 5.747 & 0.277 & 0.389 & 5.746 & 0.277 & 0.389 & 5.746 & 0.2774 & 0.389 \\
\hline$r_{h}$ & 1.053 & 0.031 & 0.162 & 1.053 & 0.031 & 0.162 & 1.053 & 0.031 & 0.162 \\
\hline$r_{f}$ & 1.053 & 0.034 & 0.110 & 1.053 & 0.034 & 0.110 & 1.053 & 0.034 & 0.109 \\
\hline \multicolumn{10}{|c|}{ Ergodic moments } \\
\hline & \multicolumn{3}{|c|}{ global } & \multicolumn{3}{|c|}{ global BL } & \multicolumn{3}{|c|}{ local } \\
\hline$N F A^{h}$ & -2.526 & 1.286 & & -4.374 & 1.116 & & -4.132 & 1.737 & \\
\hline$c_{h}$ & 0.434 & 0.034 & & 0.386 & 0.029 & & 0.403 & 0.037 & \\
\hline$\theta_{h}^{h}$ & -0.023 & 0.150 & & -0.232 & 0.125 & & -0.208 & 0.203 & \\
\hline$\theta_{f}^{h}$ & 0.585 & 0.076 & & 0.474 & 0.075 & & 0.490 & 0.100 & \\
\hline$q_{h}$ & 5.755 & 0.270 & & 5.760 & 0.274 & & 5.758 & 0.270 & \\
\hline$q_{f}$ & 5.743 & 0.293 & & 5.749 & 0.297 & & 5.746 & 0.293 & \\
\hline$r_{h}$ & 1.053 & 0.030 & & 1.053 & 0.031 & & 1.053 & 0.030 & \\
\hline$r_{f}$ & 1.055 & 0.034 & & 1.055 & 0.034 & & 1.054 & 0.034 & \\
\hline
\end{tabular}

Table 5: Comparison of model moments in the asymmetric setting with $\sigma_{Y_{f}^{i}}=2 \sigma_{Y_{h}^{i}}$, for $i=k, l$, model specification 1 , sensitivity analysis with $\sigma_{Y_{h}^{k}}=\sigma_{Y_{h}^{k}}=0.03$ and risk aversion coefficient of 15 .

Columns 'global' and 'local' refer, respectively, to the global and DS solutions when the endogenous discount factor is present. Column 'global BL' refers to the global solution when a borrowing limit is present.

Our sensitivity experiment provides us with a setting in which, for ergodic properties, the local method has become inaccurate. These inaccuracies could stem from a) the point of approximation being inappropriate, or b) from the policy functions being non-linear and inaccurate away from the point of approximation. Our findings help us uncover the potential source of these inaccuracies. Our results on 'panel simulations' versus our results on ergodic moments seem to suggest that b) is of relevance. In particular, we find that the panel simulation results of local and global method are typically very close to each, in contrast to results on ergodic model properties. This indicates that differences may emerge when the NFA position is allowed to travel far away from the approximation point, as it happens in a really long simulated series only. ${ }^{26}$

\subsubsection{The role of the approximation point}

In this section we evaluate the performance of the local DS method depending on what approximation point is used. Typically, the convention in dynamic macroeconomics is to take a local approximation around the deterministic steady state, with an understanding that most of the model dynamics are likely to be close to this rest point, so that the approximation would

\footnotetext{
${ }^{26}$ On the hand, we argue that such dissection into 'reasons for inaccuracy' a) and b) is not entirely possible, as they are interrelated. In particular, if policy functions were, in fact, truly linear, then a 'wrong' approximation point should be entirely inconsequential, and should not be a cause of differences in time paths even if the NFA drifts far away from the approximation point.
} 


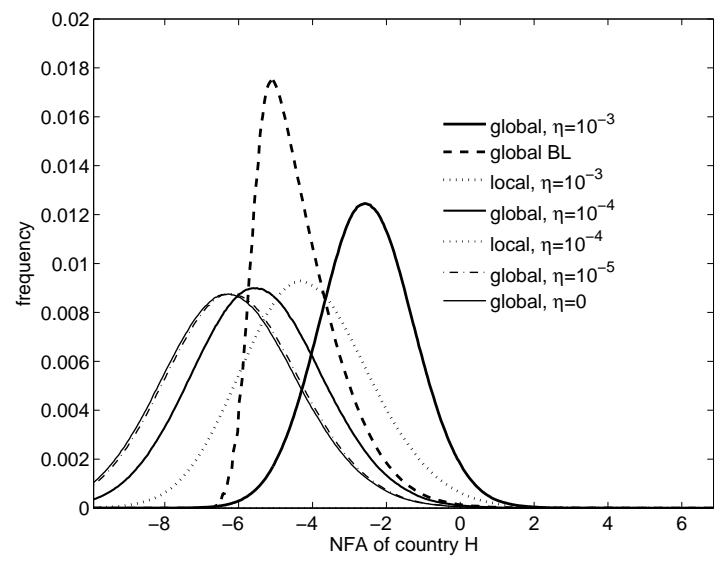

Figure 6: Ergodic distribution of NFA holdings in the asymmetric setting with $\sigma_{Y_{f}^{i}}=2 \sigma_{Y_{h}^{i}}$, for $i=k, l$, model specification 1 , sensitivity analysis with $\sigma_{Y_{h}^{k}}=\sigma_{Y_{h}^{k}}=0.03$ and risk aversion coefficient of 15 .

Labels 'global' and 'local' refer, respectively, to the global and DS solutions when the endogenous discount factor is present. Label 'global $\mathrm{BL}$ ' refers to the global solution when a borrowing limit is present.

be a 'good' description of the true nonlinear dynamics in the neighborhood of this point.

There, however, exists a recent literature that argues that the deterministic steady state may not always be the 'ideal' approximation point and that for certain applications the point of approximation matters strongly for local (perturbation) solutions (see, e.g., Julliard (2011), Coeurdacier et al. (2011, 2013), Gertler et al. (2011), De Groot (2013)). In some cases, the deterministic steady state values of the macroeconomic variables - defined as the equilibrium time-invariant values in a world of certainty - may be very different from (the mean of) the steady state (ergodic) distribution of those variables in a stochastic world. This is true, e.g., in the presence of heterogeneous agents (countries), particularly if agents are asymmetric. Moreover, in some cases the deterministic steady state is not properly defined, for example in a small open economy or in a two country incomplete markets model where equilibrium wealth is not uniquely defined, or in portfolio choice problems for which portfolios are indeterminate in the deterministic steady state. ${ }^{27}$ In such cases, Coeurdacier et al. (2011) propose to approximate the model around the so called 'risky steady state', which incorporates information about the stochastic nature of the economic environment, and which is defined to be the 'point where agents choose to stay at a given date if they expect future risk and if the realization of shocks is 0 at this point'. In practise, Coeurdacier et al. (2011) find the risky steady state as a fixed-point of an iterative method that jointly solves the steady state (which depends on second moments of model dynamics) and the dynamics of the model (which depend on the steady state). De Groot (2013) proposes a direct method, that avoids the need to resort to an iterative algorithm.

So far, the model simulations of sections 4.1 and 4.2 have followed the convention to use the deterministic steady state as the approximation point in deriving the DS solution. ${ }^{28}$

\footnotetext{
${ }^{27}$ As outlined in section 2, the DS algorithm manages to overcome the problem that steady state portfolios are indeterminate at the non-stochastic steady state. Nevertheless, the problem that the equilibrium wealth position (the net foreign asset position) is not pinned down uniquely, remains.

${ }^{28}$ This is also typically the route followed in most of the literature that has used the DS solution method
} 
It should be noted that the deterministic steady state in our model was only well defined because of the presence of the endogenous discount factor, which served as a remedy to the above mentioned problem of indeterminacy of the NFA at the deterministic steady state. In a stochastic world, the steady state distribution of NFA in our asymmetric country setting is influenced by all the factors that determine the (relative) strength of precautionary motives (such as the distribution of country specific risk faced by home and foreign households, or the menu of assets available for risk sharing), yet when looking at the deterministic steady state, we shut down exactly these forces. Instead, in the deterministic setting without stationarity inducing device there exists a whole continuum of steady state NFA position, as any $\overline{W_{h}}$ is consistent with the model's system of equilibrium conditions at the deterministic steady state. It should be noted that this problem is not specific to portfolio choice problems, it arises even when only one asset is traded and an explicit portfolio choice problem is absent, for any incomplete markets models. ${ }^{29}$ But it is potentially more consequential in a setting with non-trivial portfolio choice, since under the DS method the solution for portfolio positions obtained depends directly on the steady-state value of $\overline{W_{h}}$. While the endogenous discount factor pins down $\overline{W_{h}}$ uniquely and thus formally solves the problem of indeterminacy, it does so in a very exogenous way: the value $\overline{W_{h}}$ obtained is not based on the risk characteristics that determine the (mean of the) steady state distribution in a stochastic world. Instead it depends solely on the precise functional form of the endogenous discount factor. The value of $\overline{W_{h}}$ implied by our assumed endogenous discount factors $\left(\beta(c)=\beta c^{-\eta}\right.$ for the home country, $\beta\left(c^{*}\right)=\beta c^{*-\eta}$ for the foreign country), even in the asymmetric setting, still equals zero. ${ }^{30}$ This issue is inconsequential for models with symmetric countries where $\overline{W_{h}}=0$ is a 'natural' candidate for a steady-state. But it may pose problems in models with asymmetric countries when the ergodic distribution of $W_{h t}$ is not centered around zero. It can be argued that this approach is not more satisfactory than simply postulating the desired level of $\overline{W_{h}}$. While approximating around a well defined (unique) deterministic steady state is (because of the EDF) feasible, it is thus less clear that this value constitutes a 'good' approximation point in

(see references in footnote 2).

${ }^{29}$ Consider a one-good two-country economy. Financial markets trade only a risk-free bond. Each period countries receive a deterministic endowment and decide how much to consume and save. The equilibrium conditions of this model:

$$
\begin{aligned}
q_{b t} & =\beta u_{c h t+1} / u_{c h t}, \\
q_{b t} & =\beta u_{c f t+1} / u_{c f t}, \\
q_{b t} b_{h, t+1}+c_{h t} & =b_{h t}+y_{h t}, \\
c_{h t}+c_{f t} & =y_{h}+y_{f},
\end{aligned}
$$

determine the time paths of $c_{h t}, c_{f t}, b_{h t}, q_{b t}$, for a given $b_{h 0}$. In a deterministic steady state, all equilibrium variables are constant: $c_{h t}=\bar{c}_{h}, c_{f t}=\bar{c}_{f}, b_{h t}=\bar{b}_{h}, q_{b t}=\bar{q}_{b}, \forall t$. But at the constant values the two Euler equations reduce to the same $q_{b t}=\beta$. As a result, one is left with two independent equations for three variables $\left(\bar{c}_{h}, \bar{c}_{f}, \bar{b}_{h}\right)$ leaving us with a continuum of solutions, one corresponding to each initial bond position $b_{h 0}=\bar{b}_{h}$.

${ }^{30}$ It should be noted, that it is indeed feasible to tie down a deterministic steady state position that is different from zero. This is true, e.g., for endogenous discount factor functions $\beta(c)=\beta c^{-\eta}$ for the home country, $\beta\left(c^{*}\right)=\beta^{*} c^{*-\eta}$ for the foreign country, with $\beta \neq \beta^{*}$. The parameters $\beta$ and $\beta^{*}$ make it possible to capture structural differences in savings propensities across the two countries. For example, one could calibrate $\beta$ and $\beta^{*}$ to reach a certain desired $\overline{W_{h}}$ to match long-term averages of NFA positions in the data. Nevertheless, from a theoretical perspective, it remains unsatisfactory to base the approximation of a model around an exogenously determined steady state level of NFA, and this may lead to inaccuracies compared to the true solution. 
the sense that most of the probability mass in a stochastic world will be centered around this point in an asymmetric setting.

The concept of the risky steady state thus appears as an ideal concept to be employed as a better approximation point, in particular for the asymmetric setting of section 4.2 , when we expect that an approximation point of $\overline{W_{h}}<0$ to more accurately capture features of the true ergodic distribution of NFA. Unfortunately, the DS solution method cannot be directly amended for use at the risky steady state in an asymmetric country setting. The readyto-apply closed-form portfolio solution formulas - the reason the DS method has become popular in the literature - rely on having equal rates of return at the point of approximation. This is true both for the formula for obtaining the zero-order, steady state, portfolio (the formula given by equation (43) of Devereux and Sutherland (2011))), as well as for the formula for obtaining first-order portfolio dynamics (the formula given by equation (48) of Devereux and Sutherland (2010)). Rates of return are equal, however, only if the deterministic steady state is used as an approximation point, or, at the risky steady state for the very special case of symmetric country setups. ${ }^{31}$ In the setting where the two countries display different shock volatilities, the return of the two countries' equities will generally be different in the risky steady state.

We explore two alternatives to the approximation around the value of $\overline{W_{h}}=0$ implied by the deterministic steady state (cum endogenous discount factor), in the following. Since we found the asymmetries/ differences between local and global method to be much more pronounced for the sensitivity setting of section 4.2.1, we do so for this setting directly. The two alternatives are as follows.

One, we look at the 'stochastic steady state' implied by the second-order approximative policy functions; in this, we follow an iterative procedure that looks for an ideal approximation point $\overline{W_{h}}$, by continuously refining it using a heuristic procedure described in Devereux and Sutherland (2009). This procedure shares the principle idea with the risky steady state literature, in that the approximation point is found from the relative risk profiles of the two countries. It seems reasonable to approximate the model solution around the level of NFA that the economies tend to 'on average.' Starting at some initial approximation point

\footnotetext{
${ }^{31}$ This point is also noted by Julliard (2011), who looks at a portfolio choice model approximated around the risky steady state, but considers only a symmetric setup.

Let us formally illustrate this claim for the solution formula of steady state portfolio holdings. Solving for the steady state portfolio relies on a second order approximation to the portfolio (Euler) equations (see Devereux and Sutherland (2011)), which for the 2 equity model (assuming CRRA preferences) is given by

$$
E_{t}\left\{c_{h t+1}^{-\gamma_{h}}\left(r_{h t+1}-r_{f t+1}\right)\right\}=0
$$

Taking a second order approximation of the above expression in terms of variables in log deviations, $\widehat{x}_{t}=$ $\ln \left(X_{t} / \bar{X}\right)$, doing the same for the foreign investor's Euler equation and combining both, we get:

$$
E_{t}\left\{\begin{array}{c}
-\left(\bar{r}_{h}-\bar{r}_{f}\right)\left[\gamma_{h} \widehat{c}_{h t+1}-\gamma_{f} \widehat{c}_{f t+1}\right] \\
+\frac{1}{2}\left(\bar{r}_{h}-\bar{r}_{f}\right)\left[\gamma_{h}^{2} \widehat{c}_{h t+1}^{2}-\gamma_{f}^{2} \widehat{c}_{f t+1}^{2}\right] \\
-\left[\gamma_{h} \widehat{c}_{h t+1}-\gamma_{f} \widehat{c}_{f t+1}\right]\left(\bar{r}_{h} \widehat{r}_{h t+1}-\bar{r}_{f} \widehat{r}_{f t+1}\right)
\end{array}\right\}
$$

If and only if we have $\bar{r}_{h}=\bar{r}_{f}$, it follows that we get DS' formula from which the closed-form expression for the steady state portfolio, $\widetilde{\alpha}$, can be obtained. Whenever $\bar{r}_{h} \neq \bar{r}_{f}$, then solving for $\widetilde{\alpha}$ requires knowledge of second order solution, and the analytic formula for $\widetilde{\alpha}$ can no longer be applied.

The logic for the formula for obtaining first order portfolio dynamics is similar. Unless $\bar{r}_{h}=\bar{r}_{f}$, higher order terms appear in the third-order approximation of the portfolio (Euler) equations that precludes application of the closed order formula for first-order portfolio dynamics, $\gamma$.
} 
for the NFA ( say $\overline{W_{h}}=0$ ), we derive the NFA position that results as a rest point of the economy when it is 'hit' in every period by the mean values of the shock vector (that is, we compute the expected path of the NFA positions, as implied by the second order perturbation solution). The resulting NFA position can be used as a new approximation point and the whole procedure repeated. ${ }^{32}$ This procedure is not guaranteed to converge but it does in most cases. Applying this algorithm to the sensitivity case of our asymmetric setting of section 4.2.1, we find that the iterative procedure performs rather poorly: it converges to a net foreign asset position of $\overline{W_{h}}=-7.318$, a point that is far off from the true mean of the ergodic NFA position (-2.526 in 'global').

Two, we consider the mean of the ergodic distribution of net foreign assets obtained from having solved the model with the global method, representing the closest match to the true ergodic distribution. In the general case, in which one wants to apply the DS method to a given DSGE model, the 'true' ergodic distribution from the global solution is unknown, so this is not a feasible exercise in general. In our sensitivity asymmetric setting, the mean of the ergodic NFA distribution (for 'global') is found to be equal to -2.526 .

\begin{tabular}{|c|c|c|c|c|c|c|c|c|c|c|}
\hline & \multicolumn{2}{|c|}{ global } & \multicolumn{2}{|c|}{ global BL } & \multicolumn{2}{|c|}{$\begin{array}{c}\text { local } \\
\text { no updating } \\
\overline{W_{h}}=0\end{array}$} & \multicolumn{2}{|c|}{$\begin{array}{c}\text { local } \\
\text { with updating } \\
\overline{W_{h}}=-7.318\end{array}$} & \multicolumn{2}{|c|}{$\begin{array}{c}\text { local } \\
\text { ergodic } \mu(N F A) \\
\overline{W_{h}}=-2.526\end{array}$} \\
\hline & $\mu()$. & $\sigma()$. & $\mu()$. & $\sigma()$. & $\mu()$. & $\sigma()$. & $\mu()$. & $\sigma()$. & $\mu()$. & \\
\hline$N F A^{h}$ & 2.526 & 1.286 & 4.374 & 1.116 & -4.132 & 1.737 & -6.942 & 1.556 & -5.099 & 1.627 \\
\hline$c_{b}$ & & 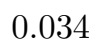 & 386 & 029 & 103 & 0.037 & 0.329 & 1 & 0. & 0.037 \\
\hline$\theta$ & -0.023 & 150 & -0.232 & 0.125 & -0.208 & 0.203 & -0.540 & 0.176 & -0.323 & 0.189 \\
\hline$\theta_{f}^{h}$ & 0.585 & .076 & 0.474 & 0.075 & 0.490 & 0.100 & 0.335 & 0.088 & 0.438 & 0.094 \\
\hline$q_{h}$ & & & & & J0 & & 年 & & & 0.270 \\
\hline $4 f$ & 5.743 & 0.293 & 5.749 & 0.297 & 5.746 & 0.293 & 5.744 & 0.293 & 5.744 & 0.293 \\
\hline
\end{tabular}

Table 6: Comparison of ergodic model moments in the asymmetric setting with $\sigma^{f}=2 \sigma^{h}$ and $\eta=0.001$, model specification $1, R A=15$.

Columns 'global' and 'local' refer, respectively, to the global and DS solutions when the endogenous discount factor is present. Column 'global BL' refers to the global solution when a borrowing limit is present.

Table 6 summarizes our findings. It presents the ergodic means and standard deviations generated using the global solution (with EDF and with BL) and the perturbation solution without $\left(\overline{W_{h}}=0\right)$, with approximation point updating $\left(\overline{W_{h}}=-7.318\right)$, and with using the mean of the ergodic NFA distribution from the global method as an approximation point $\left(\overline{W_{h}}=-2.526\right)$. Attempts at improving the performance of the DS method by searching for a more appropriate approximation point are unsuccessful.

\section{Model specification 2: a bond and an equity claim}

In model 2 we evaluate our portfolio solution methods in a specification of the general model framework of section 2 that adds further asymmetries relevant to understanding some of the key stylized facts of the international financial landscape. In particular, the asymmetry looked

\footnotetext{
${ }^{32}$ Importantly, this procedure only updates the NFA position to its 'stochastic steady state', and leaves the approximation point for all other economic variables to be equal to their deterministic steady state values. This procedure is thus consistent with application of the DS (closed form) portfolio solution formulas. We thank Alan Sutherland for laying out the details of their routine to find the approximate stochastic steady state NFA position.
} 
at in section 4.2 is not enough to provide a realistic description of financial trade between advanced versus emerging market economies. These countries are typically asymmetric in the risk they face, not only because emerging economies face higher macroeconomic volatility at business cycle frequencies, but also because their financial systems are less developed, which allows them to diversify away less of the idiosyncratic risks (see, e.g., Mendoza et al. (2009a), Caballero et al. (2006), Gourinchas et al. (2010), Maggiori (2013)). These asymmetries may be at the heart of the very asymmetric portfolio (and net foreign asset) positions observed in the data: as argued by Gourinchas and Rey (2007b, 2013), the structure and composition of portfolios of advanced economies - above all the US - shows, in contrast to emerging economies, a larger fraction of risky assets (portfolio equity or FDI) in their external balance sheets, which allows them to earn an on-average premium, or excess return, on their net position. In particular, Gourinchas et al. (2010) argue that to match this asymmetry in the structure of portfolios and to address possible expected valuation effects stemming from a systematic return differential, the modeling of different attitudes towards risk across those countries (as a short cut for a different ability to diversify domestic risk) may be essential. Model specification 2 aims to put some of these key 'finance' stylized facts at center stage. Standard macroeconomic models with CRRA preferences perform poorly in matching asset-pricing facts such as the observed equity premium. Explaining asset-pricing facts not only makes models more realistic but also increases the cost-of-business-cycles estimates and justifies policy intervention. ${ }^{33}$ It is even more important to be consistent with these facts in international macroeconomic models with portfolio choice. In the latter, asset prices determine relative wealth positions and, therefore, real allocations, and are thus important ingredients to understanding the composition of international capital flows.

Compared to model specification 1, we make two major changes in the model of this section. First, we change the menu of traded assets. We allow unrestricted trade in a oneperiod risk-free discount bond. At the same time, we assume that the equity claim of only one of the two countries is traded in the market, assumed to be the equity of the foreign economy. The budget constraint of the representative agent in country $a$ becomes:

$$
c_{a t}+\theta_{f t}^{a} q_{f t}+b_{t}^{a} q_{t}^{b}=Y_{h t}^{k}+\theta_{f t-1}^{a}\left(q_{f t}+Y_{f t}^{k}\right)+b_{t-1}^{a}+Y_{a t}^{l},
$$

While the introduction of the bond trade allows us to introduce a sharp distinction between a risky and a safe asset, the restriction on the equity trade is made for convenience only, to simplify the numerical problem for both the DS and the global solution methods ${ }^{34}$. We parameterize the model such that the risky asset earns a substantial excess return comparable in magnitude to those observed in the data ('equity premium'). Second, we allow countries to differ in their tastes towards risk, i.e. in the degrees of risk aversion. Because the investors of the two countries are heterogeneous in their tastes towards the 'higher risk' - 'higher returns' tradeoff, this naturally separates countries into equity and bond investors, as observed in the data.

In addition to being able to better match some of the key asset-pricing empirical regularities, model specification 2 allows us to test how the DS method performs in a setting where one could expect it not to perform well. Under the DS method, the main channel through

\footnotetext{
${ }^{33}$ Tallarini (2000) and Ellison and Sargent (2012) show that in the model that matches observed risk premium business cycle fluctuations are much costlier than in Lucas (1987).

${ }^{34}$ The main point of this section is to test the performance of the DS method in a setting with large risk premia. The model with one risky and one safe asset is the minimal setting which allows us to achieve this.
} 
which portfolios affect macroeconomic variables is through a multiplicative term of portfolio holdings and excess returns. ${ }^{35}$ In a setting with high risk premia, therefore, any inaccuracies in the portfolio solution, when multiplied by a sizeable excess return, may translate to higher inaccuracies also for macroeconomic variables through discrepancies in the wealthaccumulation equation. Alternatively, one may expect the local method to perform worse directly because of a difficulty to capture a sizeable excess return.

In the following, we present our evaluation of these potential concerns for a concrete parametric example, in which case the model generates an equity premium of $1.7 \%$. While, compared to model specification 1, we indeed find more substantial differences between local and global solution method, our assessment of the DS method is that it still continues to perform quite well, at least at short horizons.

Finally, we use model specification 2 to assess the importance of another dimension in which the DS method might be expected to generate inaccuracies. Rabitsch and Stepanchuk (2014) study a 2-period version of a portfolio choice problem and find that the DS method neglects the effect of the size of the shocks on the portfolio solution. As a result, the DS portfolio solution does not change as one increases the magnitude of the shocks in the model. Using the intuition from the Judd and Guu (2001) solution, Rabitsch and Stepanchuk (2014) find that the true solution generally depends on the magnitude of the shocks, with skewness, kurtosis and higher-oder moments of the risky asset returns distribution and the difference in the investors' attitude to risk affecting the results. Previewing our results, we do not find these effects to be large enough to generate sizeable discrepancies between the DS and the fully non-linear solution, so that the DS method continues to perform well in this setting as well.

\subsection{A parametric example}

\begin{tabular}{llr} 
Parameter & & Value \\
\hline Discount factor & $\beta$ & 0.95 \\
Endogenous discount factor & $\eta$ & 0.001 \\
Risk aversion & $\gamma_{h}, \gamma_{f}$ & 8,16 \\
Elasticity of intertemp. subst. & $\psi_{h}, \psi_{f}$ & 0.2 \\
Capital income share & $Y_{h}^{k} / Y_{h}, Y_{f}^{k} / Y_{f}$ & 0.30 \\
Annual mean output & $\bar{Y}_{h}, \bar{Y}_{f}$ & 1 \\
Persistence & $\rho_{Y_{h}^{k}}, \rho_{Y_{f}^{k}}, \rho_{Y_{h}^{l}}, \rho_{Y_{f}^{l}}$ & 0.80 \\
Volatility, labor inc. endow. & $\sigma_{Y_{h}^{l}}, \sigma_{Y_{f}^{l}}$ & 0.06 \\
Volatility, capital inc. endow. & $\sigma_{Y_{h}^{k}}, \sigma_{Y_{f}^{k}}$ & 0.09 \\
Correlation & $\operatorname{cor}\left(Y_{h}^{k}, Y_{h}^{l}\right)=\operatorname{cor}\left(Y_{f}^{k}, Y_{f}^{l}\right)$ & 0.50
\end{tabular}

Table 7: Parameter values, model specification 2.

Table 7 reports parameter values. We set country $h$ 's coefficient of risk aversion to 8 , and country $f$ 's coefficient of risk aversion to be twice that of country $h, \gamma_{f}=2 \gamma_{h}=16$. The intertemporal elasticity of substitution, $\psi$, in both countries is set to one fifth. The

\footnotetext{
${ }^{35}$ To be precise, and returning to our description of the DS method in section 3.2 , these are the terms $\alpha_{h t-1}^{h}\left(r_{h t}-r_{b t}\right)$ and $\alpha_{f t-1}^{h}\left(r_{f t}-r_{b t}\right)$ in equation $((16))$. Or, more precisely, in our setup of model specification 2 , where trade in domestic equity is shut down, only the term $\alpha_{f t-1}^{h}\left(r_{f t}-r_{b t}\right)$.
} 
means of the exogenous endowment processes, and the persistences remain the same as in model specification 1 . We consider a much higher volatility, though. We set the volatility of the 'labor' income shocks to $\sigma_{Y_{a}^{l}}=0.06, a=h, f$; the volatility of 'capital income' is taken to be 1.5 times higher, $\sigma_{Y_{a}^{k}}=0.09$, for $a=h, f$. This choice implies a standard deviation of aggregate $\log$-output of $\sigma\left(\log Y_{h}\right)=\sigma\left(\log Y_{f}\right)=0.060$. The correlation between a country's labor and capital income endowments is set to 0.5 . We acknowledge that in certain dimensions, particularly the size of shocks that we assume, our parameterization can be considered extreme. This is as a shortcut to generating a sizeable risk premium in this model, without resorting to an addition of features that the finance literature have deemed important recently (e.g., adding disaster shocks or long-run risk in the vain of Bansal and Yaron (2004)).

Figure 7 presents policy functions for country $h$ 's consumption share, equity holdings, bond holdings, equity price and bond price under the global solution, shown in panels A, C, E, G, I. Panels B, D, F, H and J of figure 7 show discrepancies between the global and the DS solution. ${ }^{36}$ The discrepancy for the consumption share of country $h$, equity holdings, bond holdings, equity prices, and bond prices are as large as 0.011, 0.040, 0.017, 0.013, and 0.0003 respectively. These differences are somewhat larger than in model specification 1 , but also reflect the more extreme parameterization of the current setting.

Figure 8 compares time paths from a single series of realizations of the exogenous shock process of length 100. Both methods capture the same dynamic patterns of economic variables. Compared to model specification 1, the differences between the time paths generated by the two solution methods are slightly more pronounced. In particular, the maximum difference for the consumption share, bond holdings, equity price, bond price, and NFA are, respectively, $0.179,40.230,0.190,0.050$, and 197.81 percent. The maximum absolute differences of NFA and equity holdings are 0.122 and 0.031 , respectively.

To understand these differences more systematically, it is instructive to study average simulated paths implied by the two methods. Figure 9 presents these average paths, periodby-period averages over 10000 simulations, starting each simulation run in the 'average' state with $\mathbf{Y}_{t}=E_{t}\left(\mathbf{Y}_{t}\right)$, and starting with $W_{h 0}=0$. Let us discuss the economic forces behind the evolution of average paths. Similar to the asymmetric setting in model 1, the two countries have different precautionary demands. Here, in model specification 2, the different strength of precautionary motives comes from the fact that country $f$ is more risk averse than country $h$. We should expect this channel to lead to an increase in country $f$ 's NFA position over time, or, equivalently, to a decrease in country $h$ 's NFA position. However, there is an additional channel that we should expect to impact the evolution of the NFA position over time. Our model is parameterized to display large excess returns of the risky asset over the safe asset ('equity premium'), and features a setting with heterogeneous investors, where one of the investors is more willing to trade higher risk for higher future returns. Since the less risk-averse investor invests more in the higher risk, and higher return asset, he earns the equity premium and we can expect this to positively affect his wealth position, that is, his net foreign asset position. The expected path of the NFA position of both global and local method captures this effect. The equity premium produced by global and local method are very comparable, both produce an average excess return of the risky over the safe asset of 1.7\%. These excess returns enter multiplicatively with the portfolio holdings of the risky asset in the wealth-accumulation equation. Since country $h$ 's equity portfolio holdings are

\footnotetext{
${ }^{36}$ We abstract from also presenting a version of the global solution without EDF but borrowing limits, as the insights are largely the same as in model specification 1.
} 

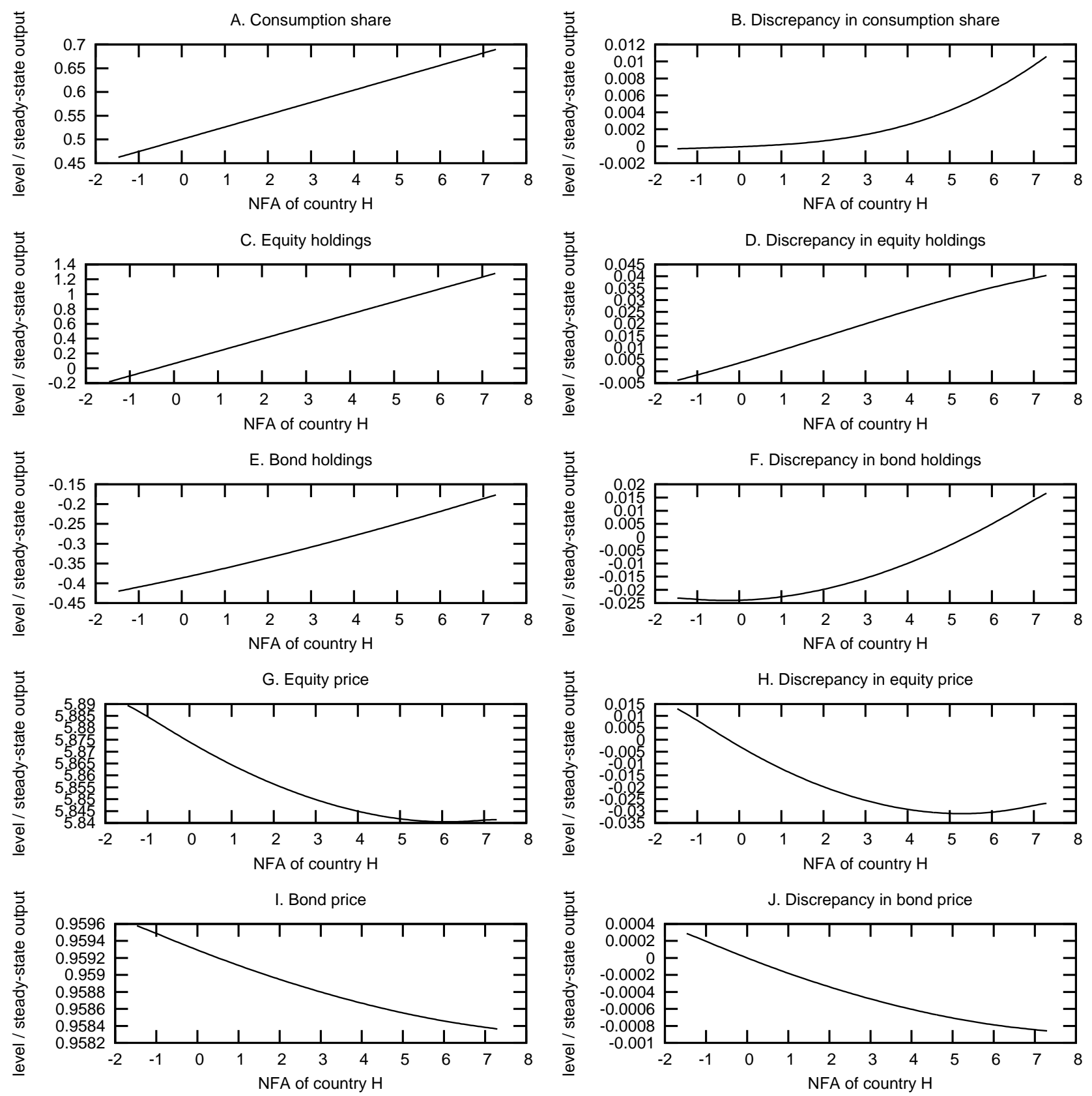

Figure 7: Country h's policy functions, model 2. Panels A,C,E,G,I present the policy functions for the global solution method. Panels B,D,F,H,J plot the discrepancy between the global and perturbation policy functions (with endogenous discount factor).

estimated to be higher under the global method than under the local, it affects country $h$ 's NFA more positively, so that at the end of period 100 this effect compounds to NFA holdings of 0.211 (21.2\% of domestic output) under the global method, which are 0.039 (3.9\% of domestic output) higher, on average, than under the local method. The difference at the end 

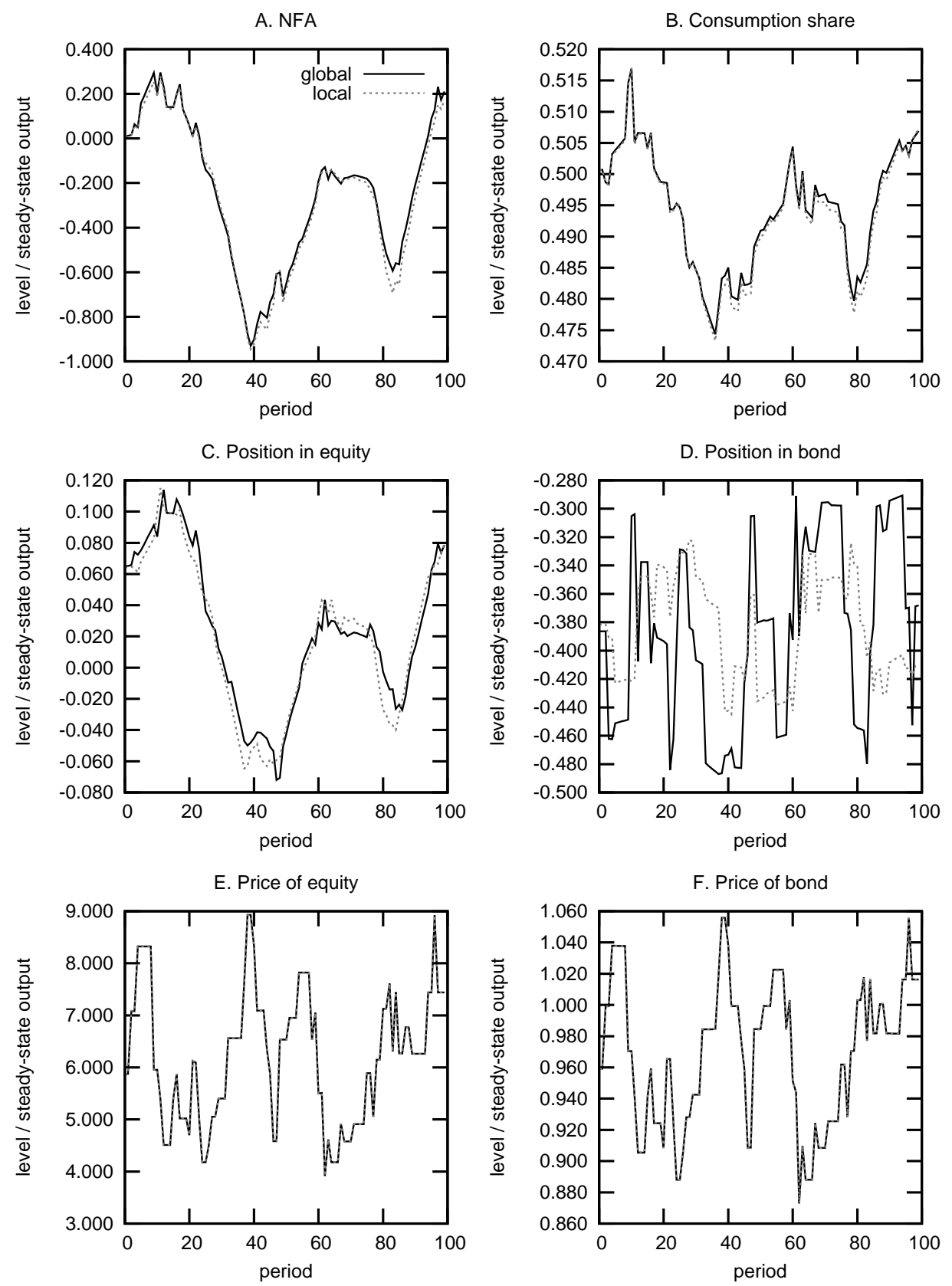

Figure 8: Single simulated time paths, model specification 2.

Labels 'global' and 'local' refer, respectively, to the global and DS solutions when the endogenous discount factor is present. 
of period 100 of the consumption share, equity holdings, and bond holdings are, respectively, $0.001,0.007$, and -0.005 . By and large, and considering that our parametric example uses volatilities of our exogenous shock processes that are on the high end, we judge that the performance of the DS method remains very reasonable.

\begin{tabular}{|c|c|c|c|c|c|c|}
\hline \multicolumn{7}{|c|}{ Moments from panel simulations } \\
\hline & $\mu()$. & $\sigma()$. & $\rho\left(., Y^{h}\right)$ & $\mu()$. & $\sigma()$. & $\rho\left(., Y^{h}\right)$ \\
\hline$N F A^{h}$ & 0.114 & 0.386 & 0.065 & 0.103 & 0.392 & 0.032 \\
\hline$c_{h}$ & 0.503 & 0.010 & 0.398 & 0.503 & 0.011 & 0.383 \\
\hline$\theta_{h}^{h}$ & 0.078 & 0.060 & -0.035 & 0.076 & 0.061 & 0.000 \\
\hline$b^{h}$ & -0.383 & 0.053 & 0.033 & -0.377 & 0.030 & -0.711 \\
\hline$q_{f}$ & 6.021 & 1.060 & 0.664 & 6.024 & 1.060 & 0.664 \\
\hline$q_{b}$ & 0.963 & 0.039 & 0.700 & 0.963 & 0.039 & 0.700 \\
\hline$r_{f}$ & 1.058 & 0.124 & 0.186 & 1.058 & 0.124 & 0.187 \\
\hline$r_{b}$ & 1.041 & 0.042 & -0.534 & 1.041 & 0.042 & -0.534 \\
\hline \multicolumn{7}{|c|}{ Ergodic moments } \\
\hline & & global & & & local & \\
\hline$N F A^{h}$ & 2.769 & 2.237 & & 7.244 & 6.205 & \\
\hline$c_{h}$ & 0.570 & 0.054 & & 0.737 & 0.202 & \\
\hline$\theta_{h}^{h}$ & 0.510 & 0.344 & & 1.267 & 1.003 & \\
\hline$b^{h}$ & -0.3135 & 0.073 & & -0.208 & 0.656 & \\
\hline$q_{f}$ & 6.006 & 1.122 & & 6.023 & 1.125 & \\
\hline$q_{b}$ & 0.962 & 0.041 & & 0.963 & 0.041 & \\
\hline$r_{f}$ & 1.058 & 0.124 & & 1.058 & 0.124 & \\
\hline$r_{b}$ & 1.041 & 0.044 & & 1.040 & 0.044 & \\
\hline
\end{tabular}

Table 8: Comparison of model moments, model specification 2.

Columns 'global' and 'local' refer, respectively, to the global and DS solutions when the endogenous discount factor is present.

Table 8 presents model moments both for 'panel simulations' (10000 series of 100 periods) and ergodic moments (from a single simulation of length 100 million). The results on panel simulations capture the same findings as our visual presentation of average paths.

To interpret these differences economically, it is again useful to look at the implied welfare consequences of these differences. Welfare differences, conditional on $\mathbf{Y}=E[\mathbf{Y}]$ and $W_{h 0}=0$, between global and local method are found to be $-19.815 \%$ and $8.913 \%$ when based on certainty equivalent consumption of the home agent or the foreign agent, respectively. These measures reflect substantially larger welfare differences compared to model specification 1 ; however, we acknowledge that they also reflect the high parameterization of shock volatilities.

The bottom part of table 8 presents simulated ergodic model moments. The difference in moments obtained from global and local method reiterate our findings from model specification 1. When the NFA position travels far from the point of approximation, as happens over a longer time period, the local approximation method becomes inaccurate. While this finding is not specific to portfolio solution methods, this means that the DS method, like any local method, should be used only with caution when one is interested in ergodic behavior of an economy. 

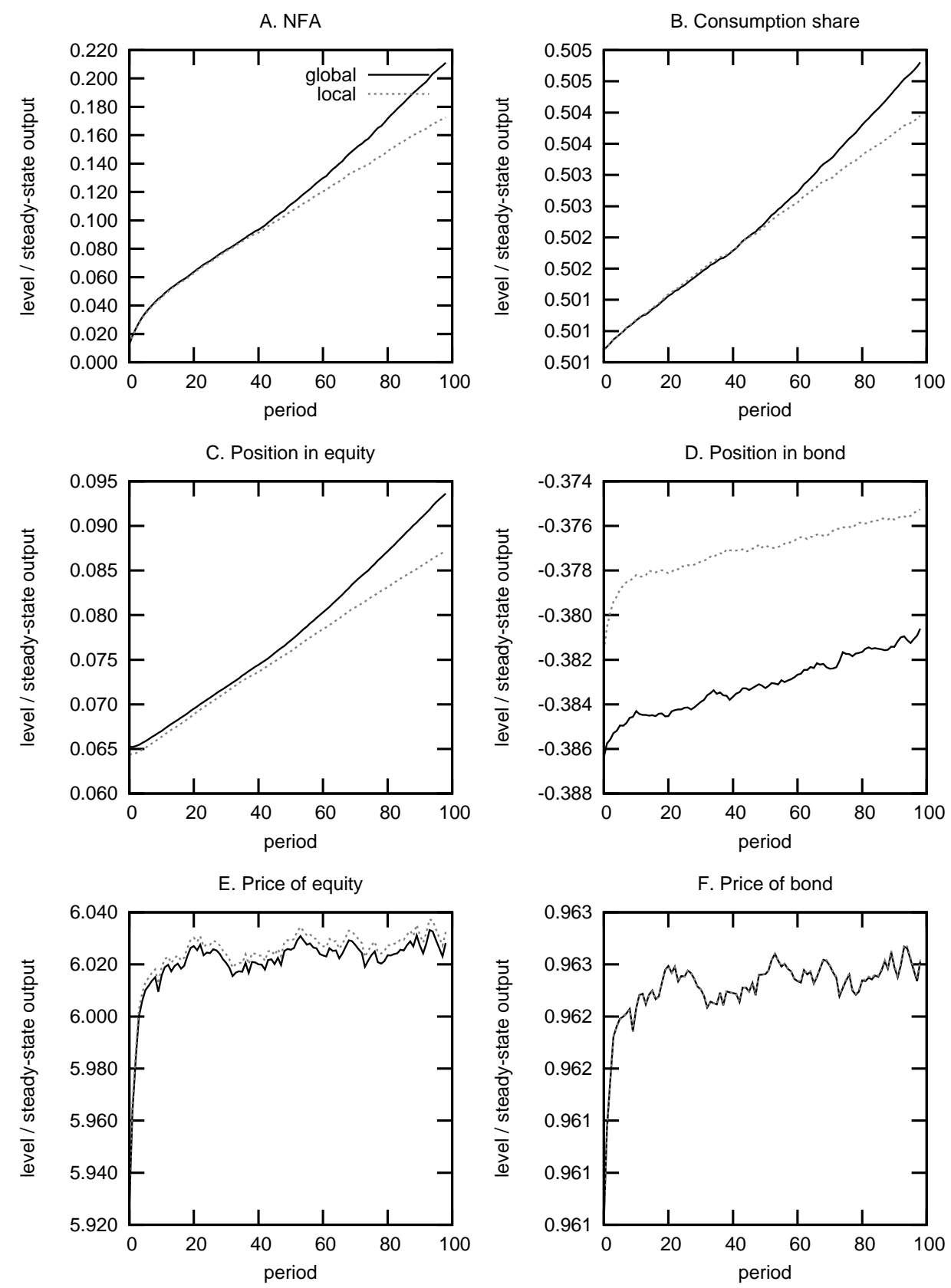

Figure 9: Average simulated paths, model specification 2.

Labels 'global' and 'local' refer, respectively, to the global and DS solutions when the endogenous discount factor is present. 


\subsection{Varying the size of uncertainty}

It is natural to expect the solution based on a local approximation method to perform worse as the size of shocks increases, since large shocks make it more likely that the model's variables will depart further away from their values at the approximation point. In addition to this, as discussed in section 3.2 on the description of the local method, the DS method neglects a (direct) dependence of the portfolio solution on the size of uncertainty. That is, the approximate portfolio solution under the DS method can be seen as a first-order expansion to the true policy function for portfolio holdings in the direction of state variables only, but ignores an expansion in the dimension of the scale of shocks. This can further compromise the performance of the perturbation-based method. To investigate this possibility, this section studies the behavior of global and DS solution as we change the size of uncertainty in the model. Figure 10 summarizes the results. For simplicity, to make changing the size of uncertainty easier, we assume in this section that all shocks have the same size, and are uncorrelated. This means that compared to table 7, we make 2 changes: (1) we set $\sigma_{Y_{h}^{l}}=\sigma_{Y_{f}^{l}}=\sigma_{Y_{h}^{k}}=\sigma_{Y_{f}^{k}}=\sigma$, and (2) set $\operatorname{cor}\left(Y_{h}^{k}, Y_{h}^{l}\right)=\operatorname{cor}\left(Y_{f}^{k}, Y_{f}^{l}\right)=0$. We then change the size of uncertainty in the model by varying $\sigma$.

Panels $\mathrm{A}$ and $\mathrm{B}$ of figure 10 show the portfolio positions in equity and bond, $\theta_{h t}^{h}$ and $b_{t}^{h}$, that correspond to an end-of-period net foreign asset position equal to 0 and exogenous shocks equal to their unconditional means $\left(W_{h t}=0, \mathbf{Y}_{t}=E_{t}\left(\mathbf{Y}_{t}\right)\right)$, as predicted by the global (solid line) and local (dashed line) solutions. Reminiscent of our findings in a two-period model in Rabitsch and Stepanchuk (2014), we find that the portfolio positions obtained from the local (DS) method do not change with the size of uncertainty, $\sigma$. On the other hand, equity holdings under the global solution decrease, and bond holdings under the global method increase, as $\sigma$ increases from 0.001 to 0.1 . This is also consistent with the results in Rabitsch and Stepanchuk (2014) and Judd and Guu (2001) ${ }^{37}$, who find that the true non-linear solution depends on the size of shocks, with higher-order moments of the distribution of the shocks such as skewness and kurtosis of the shocks (equal to the skewness and kurtosis of equity returns in that simple model) affecting the results. Panels $\mathrm{G}$ and I of figure 10 show the one-period-ahead skewness and kurtosis of equity returns at $W_{h t}=0$ and $\mathbf{Y}_{t}=E_{t}\left(\mathbf{Y}_{t}\right)$. Skewness is positive, and both skewness and kurtosis of returns increase as $\sigma$ increase. Rabitsch and Stepanchuk (2014) find that in a two-period setting with the foreign investor being more risk-averse than the domestic investor, and with positive skewness of equity returns, the first-order coefficient in an asymptotic Taylor approximation to the equity position obtained by Judd and Guu (2001) is positive, leading the domestic investor to take a larger equity position, while the second-order coefficient is negative, leading to the opposite result with the foreign investor taking a larger equity position. They also find that for a realistic calibration, using data from the United Kingdom, the second-order effect dominates. ${ }^{38}$ This is also what we get here, with the equity position of the domestic investor under the global solution decreasing as the size of the shocks, $\sigma$, goes up.

Panels $\mathrm{E}$ and $\mathrm{F}$ of figure 10 show that at $\sigma=0.1$, the equity premium in our model reaches a reasonable size of $2.8 \%$. However, $\sigma=0.1$ is quite higher than the size of shocks

\footnotetext{
${ }^{37}$ Judd and Guu (2001) develop a Taylor series approximation to the portfolio positions in a static two-period model, using a bifurcation method to solve for the approximation coefficients. Rabitsch and Stepanchuk (2014) compare this solution to the one obtained using the DS and global methods.

${ }^{38}$ Note that compared to the 2-period model case, in this paper with long-lived equity the equity returns are endogenous. This means that we cannot change their skewness and kurtosis separately.
} 
usually assumed in the dynamic macroeconomic models. Panel D of figure 10 shows that the DS solution underestimates the size of the equity return compared to the global solution. As a result, as shown in panel $\mathrm{C}$, the NFA position in the last period $(\mathrm{T}=100)$ of our short simulations on average is higher according to the global solution. Note that, as pointed out above, panels $\mathrm{A}$ and $\mathrm{B}$ of figure 10 show the portfolio positions consistent with the end-ofperiod net foreign assets equal to zero, $W_{h t}=0$. Figure B.3 in the appendix shows the portfolio positions that correspond to beginning-of-period net foreign assets equal to zero, $W_{h t-1}=0 .{ }^{39}$ While the DS solution of $\theta_{h t}^{h}$ (and $b_{t}^{h}$ ) were found to be constant with respect to changes in the size of uncertainty when plotted as a function of $W_{h t}=0$, documenting the lack of a direct dependence of the portfolio solution on $\sigma$, we observe it to decrease (increase) as $\sigma$ increases when $W_{h t-1}=0$. Similarly, while, under the global method, the equity position of the domestic investor in figure 10 decreases with $\sigma$, the one in figure B.3 is in fact increasing with $\sigma$. The portfolio positions in figure B.3 more closely correspond to the NFA(T) in figure 10 , since we always start with the beginning-of-period net foreign assets, $W_{h t-1}$, equal to zero to obtain the simulated NFA(T). However, those portfolio positions also reflect the reaction of the consumption and savings decisions to the size of uncertainty in our model. Through the dependence of state variables on the size of uncertainty, portfolio solutions are affected by variations in the scale of shocks indirectly. As a result, the DS portfolio solutions in that figure loose the 'invariance to the size of uncertainty' property which we find in the static model in Rabitsch and Stepanchuk (2014).

\section{Conclusions}

This paper compares the performance of the local portfolio solution method of Devereux and Sutherland (2010, 2011) relative to a global portfolio solution method. We present a general model framework and consider several specifications of this framework in our test suite: a symmetric country setting, standing in as an application of financial trade between advanced economies, and asymmetric country settings that reflect important features of asymmetry in financial trade, such as between advanced and emerging economies. The asymmetries looked at are differences in macroeconomic volatility, but more importantly, a setting in which one country (advanced) takes on more of risky than safe assets compared to the other other country (emerging), and earns a sizeable risk premium on its portfolio. We find, whenever we look at the behavior at short horizons, that the DS method performs very well. This is especially true in settings where assets are similar and have similar return (that is, small risk premia), whether countries are symmetric or asymmetric. We find somewhat more pronounced differences in the setting with a sizeable risk premium, because of differences in the accuracy in which the return differential affects wealth accumulation. Nevertheless, the DS method continues to work reasonably accurate also there. At long horizons, to capture ergodic features of the economy, we document that global and local method can lead to strong differences, and that for such purpose the DS method should be used with caution only.

\footnotetext{
${ }^{39}$ In our two-period model such distinction is not relevant and not applicable; the NFA position is always zero.
} 

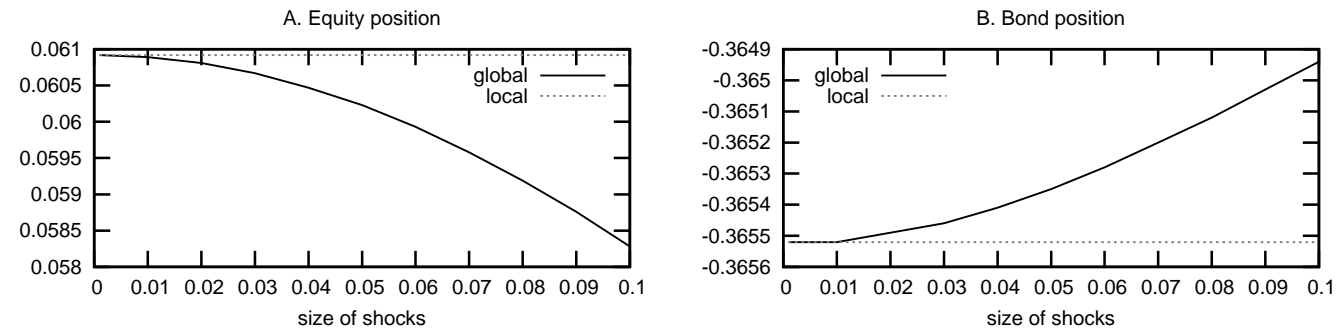

C. $\operatorname{NFA}(T)$

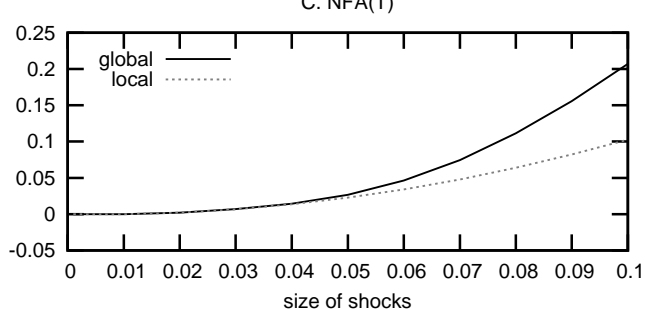

D. Discrepancy in equity premium

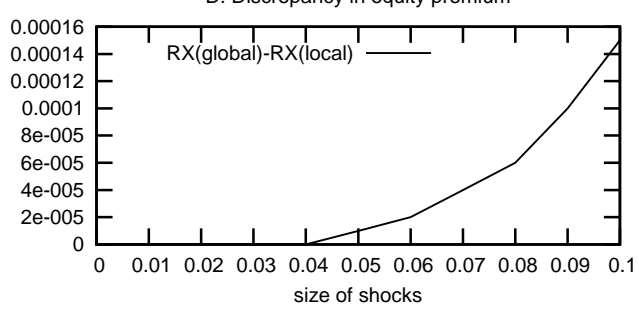

E. Mean equity returns

F. Mean bond returns
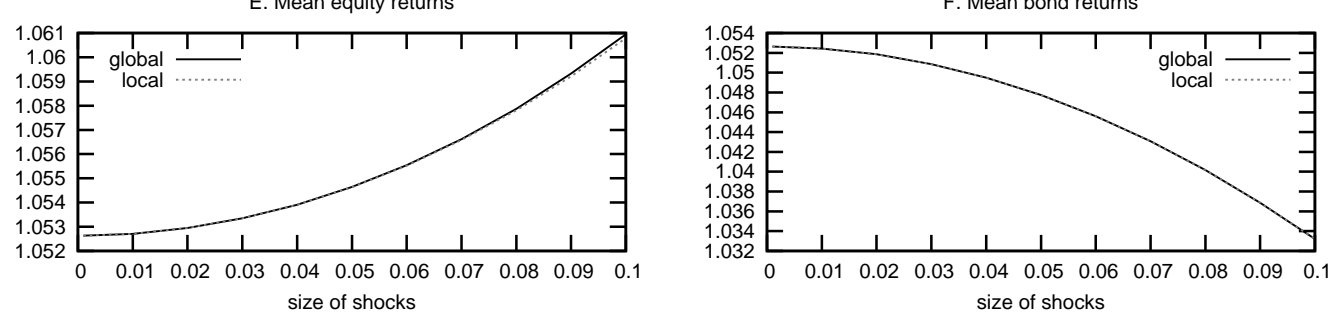

G. Equity return skewness

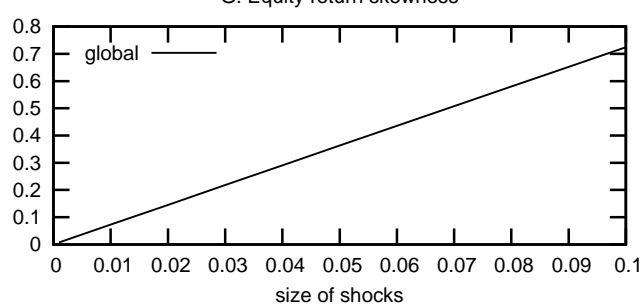

H. Equity return kurtosis

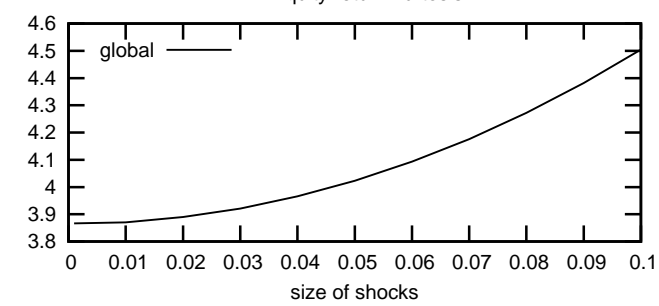

Figure 10: Varying the size of uncertainty, $\sigma$, model specification 2.

Labels 'global' and 'local' refer, respectively, to the global and DS solutions when the endogenous discount factor is present. 


\section{References}

Aguiar, M. and Gopinath, G. (2007). Emerging market business cycles: The cycle is the trend. Journal of Political Economy, 115(1):69-102.

Bansal, R. and Yaron, A. (2004). Risks for the long run: A potential resolution of asset pricing puzzles. Journal of Finance, 59(4):14811509.

Caballero, R., Farhi, E., and Gourinchas, P.-O. (2006). An equilibrium model of 'global imbalances' and low interest rates. NBER Working Paper, (11996).

Coeurdacier, N., Rey, H., and Winant, P. (2011). The risky steady state. American Economic Review, Papers and Proceedings, 101(3):398-401.

Coeurdacier, N., Rey, H., and Winant, P. (2013). Financial integration and growth in a risky world. mimeo.

De Groot, O. (2013). Computing the risky steady state of dsge models. Economics Letters, 120:566-569.

De Groot, O., Durdu, B., and Mendoza, E. (2014). Solving dsge models with incomplete markets: Global vs. local methods. mimeo.

Den Haan, W. and de Wind, J. (2009). How well-behaved are higher-order perturbation solutions? De Nederlandsche Bank Working Paper.

Devereux, M. B. and Sutherland, A. (2009). A portfolio model of capital flows to emerging markets. Journal of Development Economics, 89:181-193.

Devereux, M. B. and Sutherland, A. (2010). Country portfolio dynamics. Journal of Economic Dynamics and Control, 34:1325-1342.

Devereux, M. B. and Sutherland, A. (2011). Country portfolios in open economy macro models. Journal of the European Economic Association, 9(2):337-369.

Ellison, M. and Sargent, T. (2012). Welfare cost of business cycles in economies with individual consumption risk. Working Paper.

Evans, M. D. and Hnatkovska, V. (2005). International capital flows, returns and world financial integration. NBER Working Papers, (11701).

Evans, M. D. and Hnatkovska, V. (2012). A method for solving general equilibrium models with incomplete markets and many financial assets. Journal of Economic Dynamics and Control, 36(12):19091930.

Flodén, M. (2006). A note on the accuracy of markov-chain approximations to highly persistent $\operatorname{ar}(1)$-processes. SSE/EFI Working Paper Series in Economics and Finance, (656).

Gertler, M., Kiyotaki, N., and Queralto, A. (2011). Financial crises, bank risk exposure and government financial policy. mimeo. 
Gourinchas, P.-O. and Rey, H. (2007a). From world banker to world venture capitalist: Us external adjustment and the exorbitant privilege. In Clarida, R., editor, G7 Current Account Imbalances: Sustainability and Adjustment, pages 11-55. The University of Chicago Press.

Gourinchas, P.-O. and Rey, H. (2007b). International financial adjustment. Journal of Political Economy, 115(4).

Gourinchas, P.-O. and Rey, H. (2013). External adjustment, global imbalances, valuation effects. In Gopinath, G., Helpman, E., and Rogoff, K., editors, Handbook Chapter in International Economics, vol. IV. North-Holland.

Gourinchas, P.-O., Rey, H., and Govillot, N. (2010). Exorbitant privilege and exorbitant duty. mimeo, UC Berkeley.

Jin, H.-H. and Judd, K. (2002). Perturbation methods for general dynamic stochastic models. Manuscript, Stanford University.

Judd, K. and Guu, S. (2001). Asymptotic methods for asset market equilibrium analysis. Economic Theory, 18:127-157.

Judd, K. L., Maliar, L., and Maliar, S. (2011). Solving the multi-country real business cycle model using ergodic set methods. Journal of Economic Dynamics and Control, $35(2): 207-228$.

Judd, K. L., Maliar, L., and Maliar, S. (2012). Merging simulation and projection aproaches to solve high-dimensional problems. NBER working paper 18501.

Julliard, M. (2011). Local approximation of dsge models around the risky steady state. mimeo.

Kim, J., Kim, S., Schaumburg, E., and Sims, C. (2003). Calculating and using second-order accurate solutions of discrete time dynamic equilibrium models. DEDS Working Paper.

Kopecky, K. and Suen, R. M. H. (2010). Finite state markov-chain approximations to highly persistent processes. Review of Economic Dynamics, 13:701-714.

Kubler, F. and Schmedders, K. (2003). Stationary equilibria in asset-pricing models with incomplete markets and collateral. Econometrica, 71(6):17671793.

Lan, H. and Meyer-Gohde, A. (2013). Pruning in perturbation dsge models. mimeo.

Lane, P. (2013). Financial globalisation and the crisis. Open Economies Review, 24 (3):555580.

Lane, P. and Milesi-Ferretti, G. M. (2001). The external wealth of nations - measures of foreign assets and liabilities for industrial and developing countries. Journal of International Economics, (55):263-94.

Lane, P. R. and Milesi-Ferretti, G. M. (2007). The external wealth of nations mark ii: Revised and extended estimates of foreign assets and liabilities. Journal of International Economics, (73):223-250. 
Lkhagvasuren, D. and Gospodinov, N. (2011). A moment-matching method for approximating vector autoregressive processes by finite-state markov chains. Journal of Applied Econometrics, (forthcoming).

Lombardo, G. (2010). On approximating dsge models by series expansions. ECB Working Paper.

Lucas, R. (1987). Models of Business Cycles. Basil Blackwell Ltd., Oxford.

Maggiori, M. (2013). Financial intermediation, international risk sharing and reserve currencies. mimeo.

Mendoza, E., Quadrini, V., and Rios-Rull, J. V. (2009a). Financial integration, financial deepness and global imbalances. Journal of Political Economy, 117(3):371-416.

Mendoza, E., Quadrini, V., and Rios-Rull, J. V. (2009b). Financial integration, financial development, and global imbalances. Journal of Political Economy, 117 (3):371-416.

Rabitsch, K. and Stepanchuk, S. (2014). A two period model with portfolio choice: Understanding results from different solution methods. Economics Letters, 124(2):239-242.

Samuelson, P. (1970). The fundamental approximation theorem of portfolio analysis in therm of means, variances and higher moments. Review of Economic Studies, 37:537-542.

Schmitt-Grohé, S. and Uribe, M. (2003). Closing small open economy models. Journal of International Economics, 61 (1):163-185.

Schmitt-Grohé, S. and Uribe, M. (2004). Solving dynamic general equilibrium models using a second-order approximation to the policy function. Journal of Economic Dynamics and Control, 28:755-775.

Stepanchuk, S. and Tsyrennikov, V. (2011). International portfolios: An incomplete markets general equilibrium approach. mimeo.

Stepanchuk, S. and Tsyrennikov, V. (2014). Portfolio and welfare consequences of debt market dominance. mimeo.

Tallarini, T. (2000). Risk-sensitive real business cycles. Journal of Monetary Economics, (45):507-532.

Tauchen, G. and Hussey, R. (1991). Quadrature-based methods for obtaining approximate solutions to nonlinear asset pricing models. Econometrica, (59):371-396.

Tille, C. and van Wincoop, E. (2007). International capital flows. NBER Working Paper, 12856. 


\section{A Solution accuracy}

We evaluate the solution accuracy by computing errors in the system of equilibrium conditions on a grid of wealth with 1001 nodes. (Recall that we used only 51 node to solve the system.)

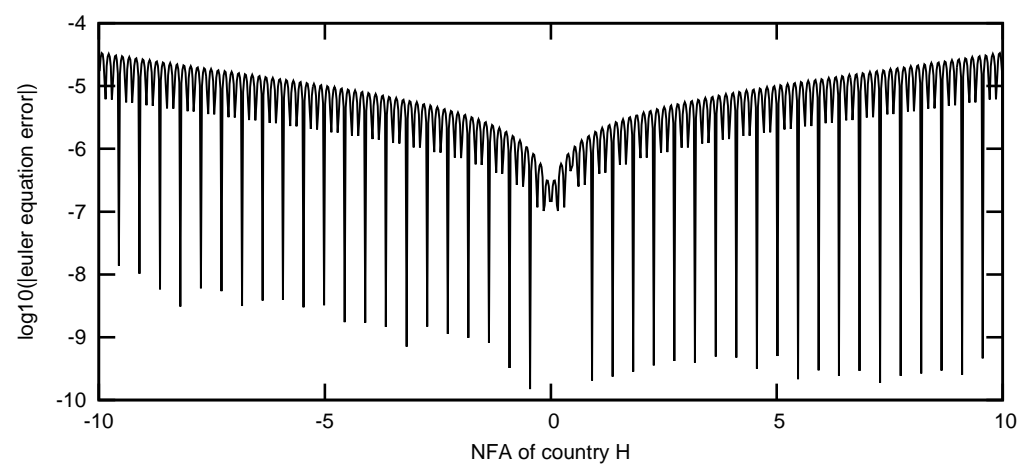

Figure 11: Equilibrium errors in the symmetric setting, model specification 1.

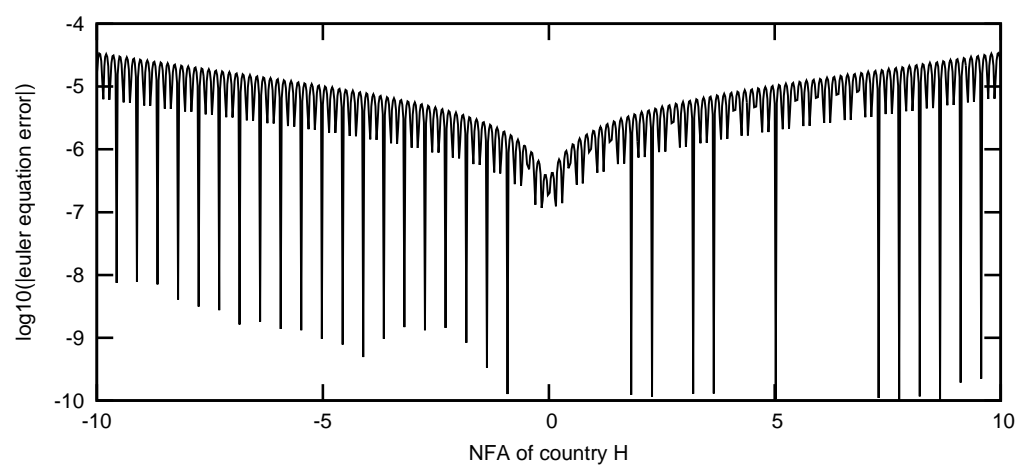

Figure 12: Equilibrium errors in the asymmetric setting with $\sigma^{h}=2 \sigma^{f}$, model specification 1 .

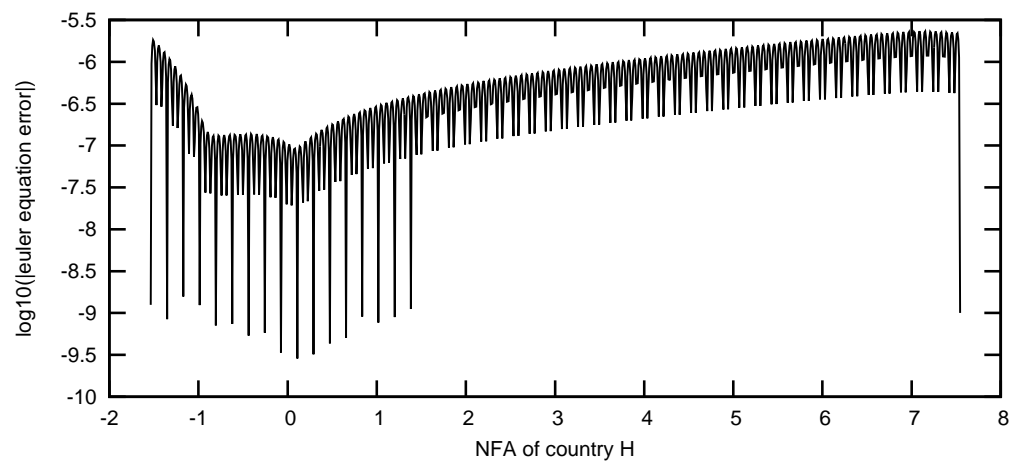

Figure 13: Equilibrium errors in model specification 2. 


\section{B Additional figures and tables}

\section{B.1 International financial integration}

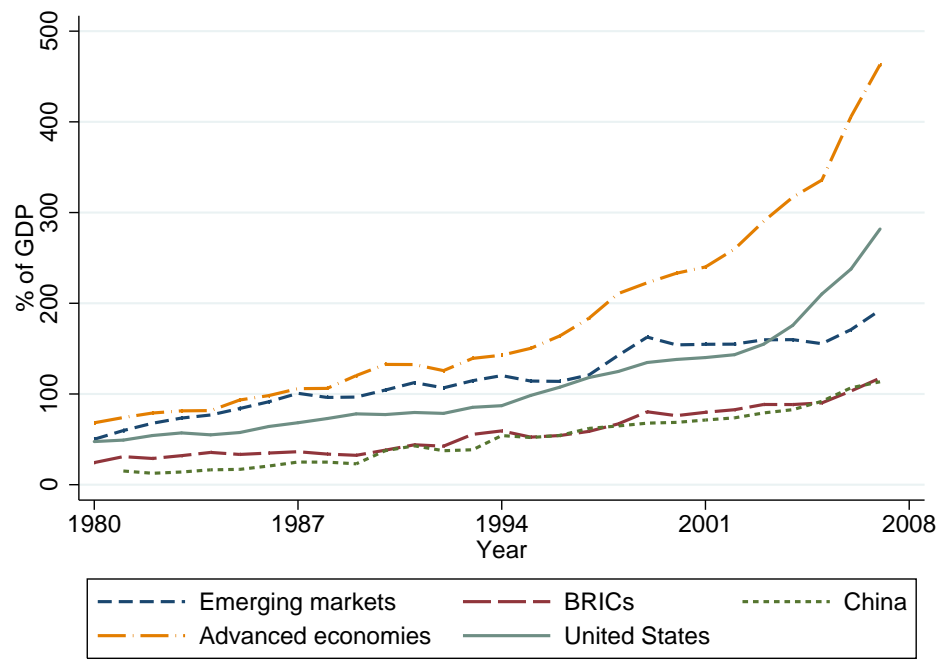

Figure 14: Gross stock of foreign assets and liabilities (data from Lane and Milesi-Ferretti [2007]), percent of country's (or country group's) GDP.

The group of advanced ecomonies comprises: Australia, Austria, Belgium, Canada, Denmark, Finland, France, Germany, Greece, Iceland, Ireland, Italy, Japan, Luxembourg, Netherlands, NewZealand, Norway, Portugal, Spain, Sweden, Switzerland, UK, US. The definition of emerging economies follows Mendoza et al. (2009b): Argentina, Brazil, Chile, China, Colombia, Czech Republic, Egypt, Hong Kong, Hungary, India, Indonesia, Israel, Jordan, Korea, Malaysia, Morocco, Pakistan, Peru, Philippines, Poland, Russia, Saudi Arabia, Singapore, South Africa, Thailand, and Turkey.

\section{B.2 Ergodic distributions of NFA}

Figure B.2 plots ergodic distributions of NFA under the global solution without endogenous discount factor but with exogeneous borrowing limits $(B L)$, for both symmetric and asymmetric settings of model specification 1 . Ergodic distributions were computed from samples of 100 million simulated observations.

\section{B.3 Portfolio positions from 'Varying the size of uncertainty' experiment of section 5.2}



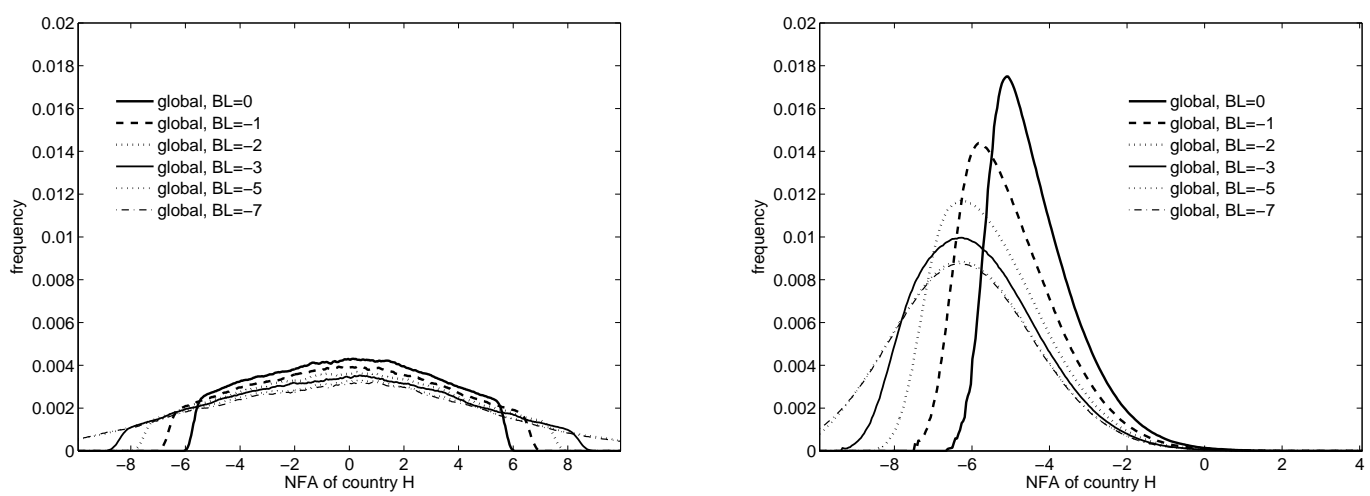

Figure 15: Stationary distributions of NFA under the global solution method with borrowing limit, for various values of borrowing limits, symmetric and asymmetric setting, model specification 1 . 

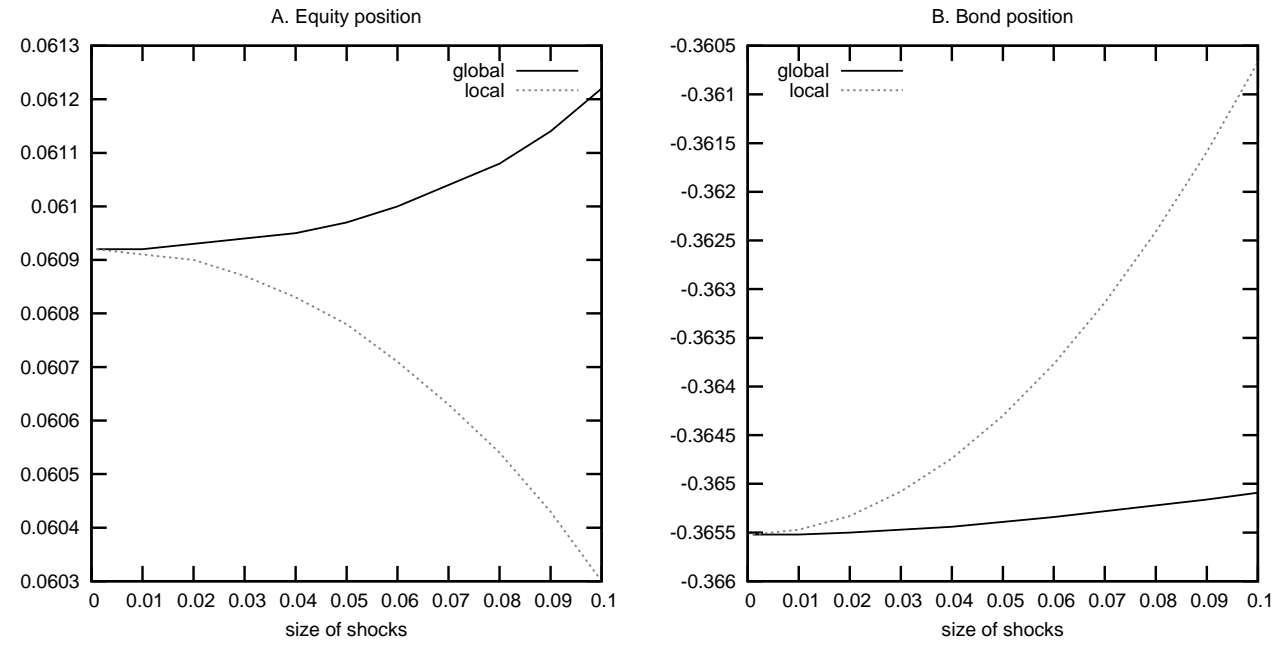

Figure 16: Portfolio solutions when varying the size of uncertainty and when beginning-ofperiod NFA, $W_{h t-1}$, equals zero, model specification 2.

Labels 'global' and 'local' refer, respectively, to the global and DS solutions when the endogenous discount factor is present. 